\section{The envelope cytoplasmic tail regulates HIV-1 assembly and spread}

\author{
Xenia Snetkov ${ }^{1}$, Tafhima Haider ${ }^{1}$, Dejan Mesner ${ }^{1}$, Nicholas Groves ${ }^{2}$, Schuyler van \\ Engelenburg ${ }^{2}$ and Clare Jolly ${ }^{*}$
}

${ }^{1}$ Division of Infection and Immunity, University College London, London WC1E 6BT, United Kingdom; ${ }^{2}$ Molecular and Cellular Biophysics Program. Department of Biological Sciences, University of Denver, Denver CO 80210, USA

* Corresponding author

Dr Clare Jolly

Division of Infection and Immunity

University College London

Gower Street WC1E 6BT

London, United Kingdom

+442081082138

c.jolly@ucl.ac.uk

The HIV-1 envelope (Env) is an essential determinant of viral infectivity, tropism and spread between T cells. Lentiviral Env contain an unusually long 150 amino acid cytoplasmic tail (EnvCT) but the function of the EnvCT and conserved domains within it remain largely uncharacterised. Here we identified a highly conserved tryptophan motif at position 757 (W757) in the LLP-2 alpha helix of the EnvCT as a key determinant for HIV-1 replication and spread between $T$ cells. Strikingly we find that mutating W757 had wide-ranging consequences including altering Env mobility in the plasma membrane, preventing Env and Gag recruitment to sites of cell-cell contact for virological synapse (VS) formation and cell-cell spread, and impeding viral fusion. Notably, W757 was also required for efficient virus budding, revealing a previously unappreciated role for the EnvCT in regulating HIV-1 assembly and egress. We conclude that W757 is a key residue that stabilises the structural integrity and 
function of Env, consistent with the recent model that this region of the EnvCT acts as a critical supporting baseplate for Env.

Keywords: HIV-1/Env/assembly/cell-cell/T-cell

\section{Introduction}

Virus assembly is a well-orchestrated event in which all components must be temporally and spatially recruited to the right place at the right time to regulate successfully infectious viral egress and spreading infection. For the lentivirus Human Immunodeficiency Virus type-1 (HIV1) virus, replication predominantly takes place in CD4+ T cells with virus assembly and budding occurring at the plasma membrane. HIV-1 has two major structural proteins: the envelope glycoprotein (Env) that is the viral attachment receptor that engages CD4 and coreceptor (CCR5 or CXCR4) and mediates fusion of the viral lipid membrane and the target cell plasma membrane to mediate entry; and the Gag polyprotein (comprising of capsid, matrix, nucleocapsid and p6) that forms the viral core. Env and Gag are trafficked independently to sites of lentiviral assembly at the plasma membrane. During HIV-1 assembly, trimers of Gag matrix (MA) assemble at the PM forming a lattice of hexamers which directly interact with phosphatidylinositol-4,5-bisphosphate (PIP2) in the membrane (Ono et al, 2004; Alfadhli et al, 2009). Recent super-resolution imaging has shown that the Gag lattice forms early during the course of viral assembly with Env arriving at a later stage (Buttler et al, 2018). Self-multimerisation of Gag, lattice associated Env, and the host cell ESCRT machinery together appear to drives virus assembly and budding (Stuchell et al, 2004; Morita et al, 2011; Prescher et al, 2015; Van Engelenburg et al, 2014). Whether the EnvCT interacts with Gag

61 MA, how exactly Env gets incorporated into virions and whether Env plays any role in regulating virus assembly remains unclear (Checkley et al, 2011; Murphy \& Saad, 2020). 
Env is composed of gp120 domain that mediates receptor binding, and the gp41 domain that is composed of an extracellular and transmembrane domain, and a long cytoplasmic tail of 150 amino acids. Conservation of the long lentiviral envelope cytoplasmic tail (EnvCT) suggests the presence of key determinants within the CT that are essential for efficient lentiviral replication and spread (Murakami \& Freed, 2000). Indeed, the importance of the EnvCT is best demonstrated by the observation that when the C-terminal EnvCT is truncated (CT $\Delta 144$ virus), HIV-1 is unable to spread in T cell lines and primary CD4 T cells due to an Env incorporation defect (Murakami \& Freed, 2000). The $\mathrm{N}$ terminus of the EnvCT consists of short membrane proximal unstructured loop containing a YxxL endocytic motif that binds the clathrin adaptor, AP-2 (Berlioz-Torrent et al, 1999; Ohno et al, 1997). Rapid endocytosis of Env from the plasma membrane via the $Y x x L$ and an additional C-terminal dileucine motif limits the amount of Env present of the surface of infected cells (Berlioz-Torrent et al, 1999; Ohno et al, 1997; Byland et al, 2007; Wyss et al, 2001; Bhakta et al, 2011). This is thought to protect infected cells from detection by the immune system and limit the amount of Env packaged into nascent virions to aid immune evasion (Stano et al, 2017; Rusert et al, 2016). Following endocytosis from the plasma membrane, retrograde and recycling trafficking pathways are proposed to coordinate Env recycling back to the cell surface (Groppelli et al, 2014; Qi et al, 2013; Wang et al, 2020; Bhakta et al, 2011; Byland et al, 2007). Previous work has shown that endocytosis mediated by $\mathrm{Y} 712$ also helps direct virus budding to the basolateral membrane in epithelial cells and also influences the site of virus budding in T cells, presumably by allowing endocytosed Env to access intracellular trafficking machinery for correct sorting back to virus assembly sites (Deschambeault et al, 1999; Groppelli et al, 2014; Kirschman et al, 2017). Thus, while the biology of the EnvCT remains to be fully-elucidated, the absolute dependence on the EnvCT for infectious virus assembly in T cells argues against a passive incorporation model and highlights the importance of the EnvCT in HIV-1 replication and spread. Notably, the first structural data of the EnvCT revealed that the majority of the long EnvCT resides buried in the membrane (Murphy et al, 2017). This is intriguing given that 90 the EnvCT of related retroviruses is considerably shorter at 30-40 amino acids (Postler \& 
91 Desrosiers, 2013). Thus, it is likely that the HIV-1 EnvCT contains additional undefined motifs

92 that regulate Env trafficking, localisation to virus assembly sites and virion incorporation.

HIV-1 spreads between CD4+ T cells via two distinct mechanisms; either by cell-free infection or by direct cell-to-cell transmission (reviewed in Sattentau, 2008). The latter is the dominant mode of HIV-1 spread (Jolly et al, 2007a, 2004; Chen et al, 2007; Sourisseau et al, 2007 ) in which the virus exploits the tight packing of CD4+ T cells in lymphoid organs and their propensity for frequent interactions to rapidly spread from one cell to another (Murooka et al, 2012). This occurs following physical contact between an HIV-1 infected donor T cells and the uninfected target T cells and is termed the virological synapse (VS) (Jolly et al, 2004). VS formation is characterised by the recruitment of HIV-1 Env and Gag to sites of cell-cell contact leading to polarised virus assembly and budding that is focused towards the target cell (Jolly et al, 2004; Hübner et al, 2009; Chen et al, 2007; Rudnicka et al, 2009). Thus, cell-cell spread is an active and regulated process that is triggered by cell-cell contact, but the mechanisms by which Env and Gag are directed to the VS and how virus assembly is orchestrated during cell-cell spread remains to be fully-elucidated. Understanding this is important, not only because may it reveal new therapeutic options to limit HIV-1 spread by targeting the latestages of HIV-1 replication, but it also provides a tractable and valuable experimental model to interrogate how HIV-1 assembly is regulated and reveal novel viral determinants.

Here we have sought to provide molecular insight into the processes of HIV-1

110 assembly and spread, specifically the role of the enigmatic EnvCT. To this end, we have

111 identified a highly conserved tryptophan residue at position 757 within the first helix of the

112 EnvCT (LLP2) that mediates HIV-1 spread between T cells, regulating Env and Gag 113 recruitment to virus assembly sites, limiting lateral diffusion of Env within the plasma 114 membrane and regulating Env fusogenecity. Thus our data identify a highly conserved site 115 within the EnvCT that plays a key role in regulating multiple Env functions, and notably reveal 116 a new and unappreciated role for the EnvCT in triggering HIV-1 assembly in T cells. 


\section{Results}

119 A conserved tryptophan residue in the EnvCT is required for HIV-1 cell-cell spread and virological synapse formation

To identify novel determinants in EnvCT that play a role in HIV-1 replication and spread we focused on tryptophan $(\mathrm{W})$ residues that possess a large bulky hydrophobic side chain and act as key mediators of protein-protein and protein-lipid interactions, including the within the W-rich HIV-1 MPER (Salzwedel et al, 1999; Muñoz-Barroso et al, 1999). The 150 amino acid HIV-1 EnvCT contains five tryptophan residues at positions 757, 790, 796, 797 and 803 (Fig. 1A). Analysis of 5916 HIV-1 Env sequences from the Los Alamos database (http://www.hiv.lanl.gov/) revealed that three of these (W757, W790, and W803) are highlyconserved amongst HIV-1 isolates (Fig. 1A) (Crooks et al, 2004; Foley et al, 2018). We focused on the two tryptophan residues at W757 and W790, given that disruption of Y802W803 has been previously characterised and shown to reduce Env incorporation and infectivity (Lambelé et al, 2007; Blot et al, 2003). Using a mutagenesis approach, the first

133 tryptophan at the beginning of the LLP-2 helix of the cytoplasmic tail of HIV-1 envelope (W757)

134 was substituted for alanine in full length replication competent HIV-1 NL4.3 and the effects on viral replication and cell-cell spread were examined. To normalise infection (and obviate any differences in the proportion of HIV-1 infected cells that may bias analysis), HIV-1 wild type (WT) and the tryptophan mutant (W757A) were pseudotyped with VSV-G to achieve equivalent initial infection of T cells. We focused on cell-cell spread that is the dominant mode of HIV-1 dissemination between T cells and takes place at the VS. Jurkat cells (donors) were

140 infected with WT or W757A virus for up to 48 hours, mixed with dye-labelled uninfected Jurkat 141 cells (targets) and cell-cell interactions and VS formation were quantified. Consistent with 142 previous reports, co-culturing HIV-1 WT infected donors and uninfected targets resulted in $14362 \%$ of cell-cell contacts forming a VS, evidenced by polarisation of Env and Gag on infected cells to the sites of contact with a target cell (Fig.1B, C). Strikingly, substituting the tryptophan

145 at position 757, with an alanine (W757A virus) abolished VS formation resulting in loss of both

146 Env and Gag recruitment to the contact zone (Fig. 1B, C). Importantly, the frequency of cell- 
147 cell contacts formed between donor and target $\mathrm{T}$ cells remained unchanged, implicating a

148 specific defect in VS formation and not an inability of cells to interact (Fig. 1D). By contrast to

149 WT Env, W757A Env displayed a more punctate, uninform distribution around the plasma

150 membrane that was not enriched at the contact zone (Fig. 1B, E). As expected, the inability of

151 the HIV-1 Env W757A mutant to form VS resulted in a significant defect in HIV-1 cell-cell spread. Co-culture of WT infected Jurkat T cells and uninfected target T cells resulted in $55 \%$ of target $\mathrm{T}$ cells becoming infected after $24 \mathrm{~h}$, by contrast to W757A virus that was unable to spread (Fig. 1F and Supplementary Fig. 1). Similar results were obtained when spreading infection was performed using primary CD4+ T cells; however, in this case the defect in W757A virus was even more pronounced, with no increase in viral spread to target cells evident up to 50hr post-infection (Fig. 1F and Supplementary Fig. 1). By contrast to the W757A mutant, the W790A mutant displayed no defect in VS formation or cell-cell spread (Supplementary Fig. 2). Therefore, we focused on understanding the specific requirement for W757 on Env biology and viral spread.

\section{Single molecule tracking of Env at virus assembly sites}

163 To explain the defect in spreading infection of W757A virus, and the inability of Env to polarise to virus assembly sites at the VS, single particle tracking (SPT) was performed on Env trimers proximal and distal to super-resolved sites of virus assembly to interrogate the mobility of wildtype EnvCT and W757A mutants (Groves et al, 2020). This approach has previously elucidated the dynamics of Env mobility at the cell surface, revealing that during nascent virus assembly Env becomes trapped or corralled in the Gag lattice to facilitate incorporation into virions (Pezeshkian et al, 2019; Buttler et al, 2018) (Fig. 2A). Importantly, the lateral diffusion of Env within and outside virus assembly sites was shown to be dependent on the EnvCT, as well as Gag MA (Pezeshkian et al, 2019). Thus, we hypothesised that failure of Env W757A to localise to virus assembly sites and polarise to the VS may be explained by a lattice trapping

173 defect, leading to inefficient incorporation of Env into the budding Gag lattice. Exploring this

174 notion, single-molecule tracking of Env and super-resolution imaging Gag assembly sites 
175 revealed that trimers of Env W757A were significantly more mobile in the PM compared to WT

176 Env, with Env W757A diffusing more freely when distal to the Gag lattice (lower panel - Fig.

$1772 \mathrm{~A}, \mathrm{~B} ; \mathrm{D}_{\text {apparent }}=0.07 \pm 0.17$ for Env WT versus $\mathrm{D}_{\text {apparent }}=0.05 \pm 0.10$ for Env $\mathrm{W} 757, P<$

$1780.0001 ; S_{M S S}=0.12 \pm 0.21$ for Env WT versus $S_{M S S}=0.13 \pm 0.22$ for Env W757, $P=0.0017$ ).

179 By contrast, proximal W757A mutant trimers exhibited similar diffusion characteristics as

180 lattice proximal WT Env trimers $\left(D_{\text {apparent }}=0.038 \pm 0.06\right.$ for Env WT $D_{\text {apparent }}=0.033 \pm 0.03$ for

181 Env W757, $P=0.0693 ; S_{M S S}=0.03 \pm 0.13$ for Env WT versus $S_{M S S}=0.05 \pm 0.16$ for Env

$182 W 757, P=0.3743$ ), suggesting that W757A mutant trimers are not defective for lattice trapping

183 and confinement (upper panel - Fig. 2B, C). Taken together these data suggest a role for

184 W757 in the EnvCT in modulating Env movement within the PM.

\section{W757 is required for efficient HIV-1 budding and infectivity}

Integrating the striking defect in cell-cell spread and VS formation with super-resolution singlemolecule imaging of Env suggested that mutating W757 in the EnvCT perturbed Env mobility in the PM and therefore the temporal and spatial recruitment of Env to virus assembly sites; however, we were struck by the observation that Gag also failed to polarise to the VS when cells were infected with the W757A Env mutant virus. This suggests a hitherto unappreciated role for the EnvCT (specifically W757) in regulating Gag-dependent HIV-1 assembly and budding from $T$ cells for subsequent viral spread. To explore this further, we quantified the cell-free budding and infectivity of virus produced by Jurkat $T$ cells and primary CD4+ T cells infected with WT or W757A virus. Strikingly, infection of both Jurkat and primary CD4+ ${ }^{+}$cells with W757A virus resulted in a significant $4-5$ fold reduction in HIV-1 budding compared to WT virus, quantified by SG-PERT assay for reverse transcriptase activity in culture supernatants (Fig. 3A). Importantly, mutating W757 did not result in amino acid changes to Rev that have been reported to mediate budding defects when the EnvCT is truncated (Durham \& Chen, 2015). Measuring virus infectivity by titrating virus-containing supernatants onto HeLa TZMbl cells and normalising the relative light units (RLU) to RT units showed that not only were

202 fewer particles released from infected T cells, but that these viral particles were significantly 
203 less infectious (Fig. 3B). However, examining Env in virions by immunoblotting showed that

204 incorporation of Env gp120 into viral particles was similar between WT and W757A virus (Fig.

$2053 C$ ) with no significant difference between these viruses in the gp120:p24 ratio in virions (Fig.

206 3D). Consistent with no observable Env incorporation defect, mutation of Q62 in the Gag MA,

207 which has been shown to rescue Env incorporation defects, did not revert the W757A mutant

208 phenotype back to WT (Tedbury et al, 2013; Ono et al, 1997)(Supplementary Fig. 3). The lack

209 of an Env incorporation defect is also consistent with the single-molecule tracking data

210 showing that both WT Env and W757A Env get trapped in the Gag lattice at the PM, and thus

211 would be expected to get incorporated into budding virions (Pezeshkian et al, 2019). Taken

212 together, these data suggest that W757 in the EnvCT is not only a determinant of Env

213 recruitment to virus assembly sites, but that it also contributes to regulation of HIV-1 budding

214 and infectivity.

215

216 Synthesis, processing, and recycling of EnvCT W757A is equivalent to that of WT

217 EnvCT

218 To address the observation that W757 in the EnvCT is required for viral infectivity, but apparently not virion incorporation, we tested Env expression, processing, localisation and trafficking in infected T cells. VS formation (and subsequent cell-cell spread) is dependent on expression of the Env glycoprotein on the surface of infected cells interacting with cell surface receptors on target cells (Jolly et al, 2004). Immunofluorescence microscopy showed no difference in intracellular staining of WT and W757A Env (Fig. 4A), with both displaying perinuclear localisation consistent with the bulk of Env residing in Golgi compartments as expected (Blot et al, 2003). Immunoblotting of infected cell lysates confirmed similar levels of unprocessed Env gp160 and the furin-cleavage products gp120 and gp41 (Fig. 4B). To compare surface expression of Env on infected T cells flow cytometry analysis was performed. WT and W757A infected T cells (Jurkat and primary CD4+ $\mathrm{T}$ cells) expressed identical levels of Env on the surface of infected cells (Fig. 4C). Once at the PM, a highly conserved membrane proximal AP-2 binding motif $(\mathrm{YxxL})$ acts to limit the amount of Env presented at 
231 the surface of infected cells with internalised Env being recycled back to the PM (Mesner et

232 al, 2020; Groppelli et al, 2014; Kirschman et al, 2017). To test the recycling kinetics of WT and

233 W757A Env we used a dual-fluorophore flow cytometry-based assay (Anand et al, 2019;

234 Mesner et al, 2020). Two Env antibodies, 2G12 and PGT151 were allowed to bind surface

235 Env at $4^{\circ} \mathrm{C}$ and detected using a PE-conjugated secondary antibody. Recycling over time was

236 allowed to proceed at $37^{\circ} \mathrm{C}$ and the population of Env that returned to the surface was detected

237 with a second Cy5-secondary resulting in double PE/Cy5 labelled Env. No significant

238 difference in recycling of endocytosed Env back to the PM was seen between WT and W757A

239 Env (Fig. 4D). Finally, we tested whether Env W757A was competent for CD4 and co-receptor

240 (CXCR4) binding and trimer formation using neutralisation assays (Montefiori, 2009). WT and

241 W757A viruses were both neutralised by bNAb PGT151 (that binds to the gp120/gp41 trimer

242 interface and detects functional Env trimers (Blattner et al, 2014; Falkowska et al, 2014)),

243 soluble CD4 (sCD4) and the CXCR4 co-receptor inhibitor AMD3100 (Donzella et al, 1998)

244 (Fig. 5A). While subtle differences in neutralisation were observed between WT and W757A

245 virus, these are insufficient to explain the striking defect in viral infectivity. In order to

246 investigate whether a fusion defect may be responsible for the decrease in Env W757A

247 infectivity, neutralisation with the fusion inhibitor Enfuvirtide (T20) was carried out. Strikingly

248 Env W757A was unable to be completely neutralised with T20. Compared to WT Env, a

249 maximum inhibition of $50 \%$ was achieved (Fig. 5A). To test whether W757 was fusion-

250 competent the BlaM-Vpr fusion assay was used to measure the capacity of Env W757A virions

251 to fuse with target cells, resulting in BlaM-Vpr release into the target cell and cleavage of CCF2

252 (Cavrois et al, 2002). Fig. 5B shows that Env W757A was significantly less able to mediate

253 virion fusion compared to WT virus, demonstrating that the infectivity defect of W757A can be

254 explained by a defect in fusion of the viral and host cell membranes and thus reduced virus

255 entry (Fig. 5B).

256

257 Conservation of W757 across different HIV lineages and ancestors 
258 Finally, we sought to explore conservation of W757 across HIV-1 groups and related

259 lentiviruses. Examining 194 Env sequences at position 757 across HIV-1 group M, N, O, P,

260 SIVgor and SIVcpz using Chromaclade (Monit et al, 2019; Foley et al, 2018) illustrated that a

261 W at position 757 is highly conserved amongst HIV-1 group M, SIVcpz and HIV-1 group N;

262 but not group O, or HIV-2 and the related SIVsmm and SIVmac viruses (Fig. 6A and

263 Supplementary Fig. 4). Noting that all HIV-1 sequences (M, N, O and P) contained an aromatic

264 residue at 757 ( $\mathrm{W}$ or $\mathrm{Y}$ ) we hypothesised that conservation of an aromatic residue at this

265 position was important and that substituting the $A$ for a $Y$ or $F$ would rescue virus replication

266 and spread. Indeed, substituting the A at position 757 with a $\mathrm{Y}$ (HIV-1 O group) or F (another

267 aromatic) rescued the W757 mutant and phenocopied the WT W757 virus, restoring virus

268 budding (Fig. 6B), infectivity (Fig. 6C) and cell-cell spread (Fig. 6D). Taken together this data

269 implicate evolutionary conservation of an aromatic residue in the EnvCT at position 757 as a

270 potentially important feature of HIV-1.

271

272

Discussion

273 Here we have uncovered a key role for a highly-conserved tryptophan residue (W) in the HIV-

2741 EnvCT that is essential for correct temporal and spatial regulation of HIV-1 assembly

275 following cell-cell contact and subsequent viral spread between T cells. The mechanism by

276 which HIV-1 assembly is regulated, and particularly the spatiotemporal events governing Env

277 and Gag recruitment to the contact zone during synapse formation leading to polarised viral

278 assembly remain largely unclear. Moreover, why lentiviruses conserve such an unusually long

279 EnvCT and what role it plays on HIV-1 assembly and infectivity remains enigmatic. Given the

280 dominance of highly-efficient cell-cell spread in viral dissemination, understanding how this

281 process is coordinated is important (Jolly et al, 2004; Chen et al, 2007; Sourisseau et al, 2007).

282 Multiple (but not mutually exclusive) mechanisms for Env and Gag recruitment to sites of virus

283 assembly at the VS have been proposed. In the case of Env, there is evidence for Env being

284 directed to the VS via lateral diffusion, outward transport from polarised secretory apparatus 
and recycling (Wang et al, 2020; Kirschman et al, 2017; Starling et al, 2016). By contrast, Gag is thought to be recruited independently of Env with evidence for lateral diffusion (Hübner et al, 2009) and polarised trafficking (Groppelli et al, 2015) to the contact zone. By coupling viral assays with single particle tracking at superresolved virus assembly sites we find that the EnvCT via W757 is required to direct Env and Gag to sites of cell-cell contact and mediate VS formation and spreading infection in T cells. Notably, single-molecule tracking revealed that mutating W757 resulted in Env diffusing more freely within the PM compared to WT Env.

292 Structural insights of the EnvCT by solution state NMR revealed that much of the EnvCT associates closely with cellular membranes, and W757 at the start of the first alpha helix (LLP-

294 2) in the EnvCT appears to provide a crucial anchor point in order to regulate membrane diffusion (Supplementary Fig. 5) (Murphy et al, 2017; Piai et al, 2020). Perturbation of this W757 anchor point may lead to changes in the quaternary structure of the putative LLP-2 baseplate and could explain an increase in PM diffusivity of the W757A mutant Env trimer compared to the native EnvCT (Piai et al, 2020). Disruption of the EnvCT baseplate by W757A may not just affect membrane diffusion, but could also alter interactions of the EnvCT with 300 host cell proteins or underlying cytoskeleton machinery that are required for polarised recruitment of Env to the VS (Jolly et al, 2004). The increased diffusion of W757A Env provides a plausible explanation for decreased W757A Env at the VS and suggests that the polarisation of Env to sites of cell-cell contact is not mediated simply by immobilising surface-associated

304 Env following binding to CD4 on target cells, but rather that cell-cell contact must specifically trigger or signal Env recruitment to the contact zone. Consistent with this notion, W757A was competent for Env-CD4 and co-receptor binding by neutralisation assay. We also found that

307 the mobility of this mutant at sites of virus assembly was unperturbed when compared to WT

308 Env trimers, suggesting that conformational changes in the baseplate structure or membrane association of LLP-2 does not impact Env incorporation and retention in the Gag lattice. This

310 is consistent with our biochemical observations that W757A showed no incorporation defect,

311 demonstrating that once Env associates with the Gag lattice it will be incorporated into viral 312 particles. Of note, while our data indicate that the earliest events of VS formation and cell-cell 
313 assembly of HIV-1 require correct and regulated Env movement within the plasma membrane,

314 they do not exclude that subsequent Env recruitment may be mediated by additional 315 mechanisms described above.

316 Env is known to be a key driver of VS formation and cell-cell spread and inhibiting Env-

317 receptor interactions abolishes VS formation (Jolly et al, 2004). We now show that the

318 contribution of Env is more than mediating stable cell-cell interactions and providing adhesive

319 interactions, rather the EnvCT appears to signal in some way to regulate the recruitment of

320 Gag to the VS, thus to control the timing and location of virus assembly. Mechanistically, these

321 observations may be explained by two possibilities. Firstly, they may allude to a sequence of

322 assembly events in which the EnvCT in the PM initiates Gag lattice formation and budding

323 following contact with a susceptible target cell (Hübner et al, 2009). In fact, the EnvCT YxxL

324 mutant has been reported to affect the site of virus budding in polarised epithelial cells and T

325 cells (Deschambeault et al, 1999; Lodge et al, 1994), supportive of an unappreciated role for

326 the EnvCT in regulating viral assembly. A second possibility is that EnvCT at the neck of the

327 budding virions is essential to complete the budding process (Buttler et al, 2018) and that

328 mutating a key residue the EnvCT inhibits this; however, while this may explain the defect in

329 cell-free viral budding, it does not explain the dramatic lack of Gag polarisation to the VS. The

330 initial engagement of Env and CD4/coreceptor results in polarisation of the cellular

331 cytoskeleton to the contact site and disrupting the actin cytoskeleton inhibits VS formation as

332 well as plasma membrane virus assembly platforms (Rudnicka et al, 2009; Chen et al, 2007;

333 Jolly et al, 2004, 2007b). Whether the underlying host cell cytoskeletal or associated cellular

334 proteins link the EnvCT to polarised Gag lattice formation in some way (via undefined host

335 factors) is an interesting possibility. It cannot be excluded that direct interactions between the

336 EnvCT and Gag take place; however, while evidence for a direct interactions have been shown

337 biochemically in vitro (Cosson, 1996; Alfadhli et al, 2019) they have not been convincingly

338 demonstrated consistently, and we were unable replicate this during the course of this study.

339 Clearly, further work is needed to determine the molecular interactions that allow the EnvCT

340 to regulate Gag localisation during viral egress. 
In addition to a lack of VS formation, cell-cell spread was also affected by a defect in

342 infectivity of the W757A mutant virus. This was initially surprising, given that many other

343 features of Env W757A remained unchanged, and unlike EnvCT truncation (CT $\Delta 144)$ that also

344 abrogate cell-cell spread (Durham \& Chen, 2015; Murakami \& Freed, 2000; Checkley et al,

2011), EnvCT W757A had no incorporation defect and did not display altered cell surface expression or recycling in T cell lines. Further analysis revealed that this infectivity defect was explained by the W757A virus showing a striking defect in fusion and thus viral entry, indicating that a single residue change in LLP-2 distal to the fusion peptide significantly impacted fusion domain structure and function. In support of our data, it has been reported that the LLP-2 helix influences fusogenicity and that viruses containing EnvCT truncations are less sensitive to T20 inhibition (Wyss et al, 2005; Kalia et al, 2003; Lu et al, 2008). Recent structural insights of the EnvCT have identified the role of the LLP-2 helix to act as a baseplate that supports the Env transmembrane domain (TMD) and stabilises the MPER region of Env, holding both in the correct structural antigenic state (Piai et al, 2020). It is highly plausible that W757 in LLP2 is key to maintenance of the EnvCT baseplate (Supplementary Fig. 5). Mutation of the hydrophobic W757 may be sufficient to perturb the baseplate, conferring conformational changes via the TMD (Piai et al, 2020) on the MPER and Env fusion domains, destabilising the Env ectodomain leading to reduced Env fusogenicity. Thus, our data support with the notion that the EnvCT baseplate plays a putative role in regulating viral entry (Piai et al, 2020). Of note, retroviruses that contain shorter EnvCTs like MLV exploit a C-terminal R peptide that controls membrane fusion activity, deletion of which lowers Env fusogenicity (Kubo et al, 2007). Thus it appears a key function of the long lentiviral EnvCT and its membrane associated structure may be to regulate fusion, and in the case of HIV-1, via LLP-2 and specifically residue W757.

In conclusion, here we have identified a single tryptophan residue in HIV-1 EnvCT that potently regulates HIV-1 assembly, virion fusion and cell-cell spread between T cells. That this suggests to a fundamental role for conservation of this reside in HIV-1 replication. This may 
prove a promising future therapeutic target to interfere with HIV-1 assembly and inhibit the dominant mode of HIV-1 dissemination.

\section{Materials and Methods}

\section{Cells and viral constructs}

Jurkat CE6.1 T cells were grown in RPMI 1640 medium (Thermo Fisher Scientific)

supplemented with $10 \%$ fetal calf serum (FCS, Labtech) and $100 \mathrm{U} / \mathrm{ml}$ Penicillin-Streptomycin

376 (Thermo Fisher Scientific). HEK 293T and HeLa TZM-bl cell lines were grown in DMEM medium (Thermo Fisher Scientific) supplemented with 10\% FCS, $100 \mathrm{U} / \mathrm{ml}$ Penicillinhealthy donors using Ficoll Paque Plus (Sigma) according to manufacturer's instructions. CD4+ T cells were isolated by negative selection using MojoSort Human CD4 T Cell Isolation kit (Biolegend). Primary CD4+ T cells were maintained in complete RPMI 1640 medium supplemented with $10 \mathrm{IU} / \mathrm{ml} \mathrm{IL-2} \mathrm{(Center} \mathrm{for} \mathrm{AIDS} \mathrm{Reagents} \mathrm{[CFAR],} \mathrm{National} \mathrm{Institute} \mathrm{of}$

383 Biological Standards and Controls, UK). Primary CD4+ T cells were activated for 4-5 days on T25 flasks coated with $5 \mu \mathrm{g}$ anti-CD3 antibody (clone OKT3, Biolegend) in the presence of $2 \mu \mathrm{g} / \mathrm{ml}$ soluble anti-CD28 antibody (clone CD28.2, Biolegend). The CEM-A cell line was obtained through NIH AIDS Reagent Program, Division of AIDS, NIAID, NIH: CEM-A from Dr. Mark Wainberg and Dr. James McMahon, CEM-CL10 (Tremblay et al, 1989). Complete growth media for the CEM-A cell line was prepared by combining $10 \%$ fetal bovine serum (Corning; Corning, NY, USA), 2 mM L-glutamine (Corning), 1\% hypoxanthine, thymidine (HT) solution (Corning), and Penicillin-Streptomycin (Corning) into Roswell Park Memorial Institute

391 (RPMI) medium (Corning). HIV-1 NL4.3 (donated by Dr. Malcolm Martin, obtained from

392 CFAR). Site directed mutagenesis was carried out on full length replication competent pNL4.3

393 by 15 amplification cycles using Pfu polymerase with the following primers; EnvCT W757A

394 (GTGAACGGATCCTTAGCACTTATCGCGGACGATCTGCGGAGCCTGTG), W757Y 
(GTGAACGGATCCTTAGCACTTATCTTCGACGATCTGCGGAGCCTGTG), and MA Q62R

(GACAAATACTGGGACGGCTACAACCATCCCTTCAG), followed by Dpn1 (NEB) digest and

(Promega). After 48h, virus was harvested and infectivity assayed by serial titration on HeLa TZM-bl reporter cells using Bright-Glo (Promega).

401

\section{Antibodies}

403 Antibodies used for flow cytometry: anti-HIV-1 p24-PE (KC57-RD1 Beckman Coulter); antiHIV-1 Env clone PGT151 (gift from Laura McCoy, UCL); Secondary antibody: anti-Human IgG Cy5 (polyclonal Bethyl). Antibodies for western blotting: anti-HIV-1 gp120 rabbit antisera (donated by Dr S. Ranjibar and obtained from the CFAR); anti-HIV-1 gp41 Mab 246-D (donated by Dr S. Zoller-Pazner and Dr M. Gorny, obtained from the CFAR); and anti-HIV-1 antibodies: anti-Rabbit IgG (ab216773, Abcam), anti-Mouse IgG (ab216775, Abcam) and antiHuman IgG (926-32232, Licor). The BG18-QD625 antibody fab fragment probe was produced recombinantly for single particle tracking as previously described (Groves et al, 2020).

\section{HIV-1 infections and cell-cell spread assays}

414 T cells were infected with 50,000 TCID50/million cells with VSV-G pseudotyped HIV-1 by

415 gravity infection for 4 hours. For cell-cell spread assays HIV-1 infected T cells (donors) were analysed for Gag expression by flow cytometry $48 \mathrm{~h}$ post-infection (p.i.). Uninfected T cells

417 (targets) were labelled with $2.5 \mu \mathrm{M}$ Cell Proliferation Dye eFluor 450 (Thermo Fisher Scientific) according to manufacturer's instructions. HIV-1 infected Gag+ donors and dye-labelled targets were mixed in $1: 1$ ratio, incubated at $37^{\circ} \mathrm{C}$ for indicated time points and analysed by flow FlowJo software. 
HIV-1 infected T cells were incubated for $24 \mathrm{~h}$ and virus-culture supernatants were harvested.

Total virus released into the supernatant was measured by qPCR to quantify the supernatant reverse transcriptase (RT) activity using SG-PERT assay (Pizzato et al, 2009) and RT activity was normalised to the number of Gag+ (HIV-1 infected) cells. Virion infectivity was determined by luciferase assay using HeLa TZM-bl reporter cells. To determine particle infectivity, RLU infectivity values were normalized to RT activity.

\section{Viral Fusion Assay}

NL4.3 WT or W757A was co-transfected with BlaM-Vpr (Addgene) and pAdVAntage (Promega) into 293T cells to generate virions packaging BlaM-Vpr. Virus was concentrated by ultracentrifugation and quantified by SG-PERT. Next, $10^{11} \mathrm{mU}$ RT of virus was used to infect target $10^{6}$ Jurkat cells for 4 hours by gravity infection and the BlaM-Vpr assay was performed as described (Cavrois et al, 2002).

\section{Immunofluorescence}

439 Uninfected Jurkat cells (target cells) were labelled with $10 \mu \mathrm{M}$ CellTracker Blue CMAC dye

440 (Thermo Fisher Scientific) according to the manufacturer's instructions. Infected Jurkat cells

441 (donor cells) were mixed with target cells in a 1:1 ratio and incubated with (nonblocking) anti-

442 Env antibody for $1 \mathrm{~h}$ at $37^{\circ} \mathrm{C}$ on poly-L-lysine-coated coverslips (Jolly et al, 2004). Cells were

443 fixed with $4 \%$ paraformaldehyde, permeabilised with $0.1 \%$ Triton X-100 and stained with anti-

444 Gag serum. Primary antibodies were detected with appropriate fluorescent secondary antibodies. Coverslips were mounted with ProLong Gold Antifade mounting solution (Thermo

446 Fisher Scientific). Images were acquired on a DeltaVision Elite image restoration microscope

447 (Applied Precision) with softWoRx 5.0 software. Envelope enrichment at the VS was 448 measured by the increase in fluorescence intensity at the contact site over intensity at a distal 449 membrane region, normalised to background intensity. Image processing and analysis was 450 performed using FIJI software (Schindelin et al, 2012). 


\section{Simultaneous Super-resolution and Single-Particle Tracking of HIV-1 Biogenesis}

453 All imaging, processing and analysis was done per the optimised methodology of Groves et.

454 Al., 2020 (Groves et al, 2020). Briefly, CEM-A T cells in complete RPMI medium (10\% fetal

455 bovine serum; $2 \mathrm{mM}$ L-glutamine; 1\% penicillin/streptomycin, and $1 \%$

456 hypoxanthine/thymidine; Corning, USA) were seeded on $25 \mathrm{~mm}$ No. 1.0 glass coverslips

457 (Warner Instruments; Hamden, CT, USA) and infected with VSV-G pseudotyped virus packaged with the pSV-NL4-3 HIV-1 reference genome, encoding the previously described CA-Skylan-S nanobody probe, with deletions in the gag-p6 late-domain, pol, vif, vpr, and nef

460 genes (Groves et al, 2020). Forty-eight hours post-infection, cells were stained with BG18-

461 QD625 probe and coverslips were transferred to specimen holders and mounted on a custom-

built ring-TIRF microscope with a live-cell chamber maintaining $37^{\circ} \mathrm{C}$ and $5 \% \mathrm{CO}_{2}$. Skylan-S and QD625 were simultaneously excited with a $473 \mathrm{~nm}$ laser and fluorescence was detected by separation of wavelengths using a dichroic beamsplitter housed in a W-View Gemini image splitter (Hamamatsu, Hamamatsu City, Japan). As previously described, images were focused onto two halves of a liquid cooled ORCA Fusion scientific-CMOS camera (C14440-20UP) streaming at $100 \mathrm{~Hz}$ (Groves et al, 2020). Raw images were corrected for non-uniform pixel offset and split based on wavelength. Single-molecule localisations for both channels were fit using custom software (IDL, Harris Geospatial; Broomfield, CO, USA), and lateral chromatic aberration between channels was corrected using a high density fiducial map collected after data acquisitions (100 nm TetraSpeck; Thermo Fisher Scientific). Single-molecule localisations were passed to an automated package written in MATLAB (Natick, MA). This software determined Gag assembly centroids and linked single molecule Env trajectories from

474 frame to frame. Tracks were split into 'proximal' and 'distal' classifications based on the proximity of their localisations to Gag bud centroids as previously described (Groves et al, 2020); Linking parameters: 50 ms maximum time gap, 10 localisations per track minimum, 1 pixel [108.33 nm] maximum frame-to-frame distance). The apparent trajectory diffusion

478 coefficients ( $D_{\text {apparent }}$ ) and the slope of the moment scaling spectrum ( $S_{M S s}$ ) were computed by 479 fit to a linear regression and weighted by localisation uncertainties. 


\section{Envelope Recycling Assay}

482 Infected Jurkat cells were stained with Envelope specific antibodies PGT151 Ab (5 $\mu \mathrm{g} / \mathrm{mL})$, 483 and $2 \mathrm{G} 12(5 \mu \mathrm{g} / \mathrm{mL})$ for $60 \mathrm{~min}$ at $37^{\circ} \mathrm{C}$ in FCS supplemented RPMI to allow for Env 484 internalisation (Mesner et al, 2020). Cells were thoroughly washed in ice cold FACS wash 485 buffer and surface Env was detected with anti-human-PE secondary antibody for 30 min on 486 ice to measure Env surface levels at $T_{0 \min }$. Cells were again washed extensively in ice cold buffer and incubated with anti-human-Cy5 secondary antibody for 0 to $180 \mathrm{~min}$ at $37^{\circ} \mathrm{C}$ in FCS supplemented RPMI to label Env that is recycling back to the cell surface. Cells were fixed in $4 \%$ paraformaldehyde and analysed by flow cytometry.

\section{Flow cytometry}

492 T cells were washed and incubated with antibodies (described above) at $4^{\circ} \mathrm{C}$ for surface staining and cell viability dye Zombie UV (Biolegend). Primary antibodies were detected with anti-human IgG secondary antibody, fixed and analysed. To stain for intracellular antigens, cells were fixed with $4 \%$ paraformaldhyde and permeabilised with CytoPerm buffer

496 (Biolegend). Cells were analysed on BD LSR Fortessa X-20 cytometer. Compensation was performed using CompBeads (BD) and calculated by FACSDiva software. Data was analysed using FlowJo software.

\section{Western Blotting}

501 Fifty $\mu \mathrm{g}$ of cell lysate and an equivalent volume of purified virus (purified by ultracentrifugation over sucrose) were separated by SDS-PAGE and analysed by western blotting using the

503 following primary antibodies: anti-HIV-1 gp120, anti-HIV-1 gp41, anti-HIV-1 Gag, and anti-

504 tubulin (described above). Primary antibodies were detected with appropriate fluorescent secondary antibodies and imaged with Odyssey Infrared Imager (Licor). Immunoblots were analysed with Image Studio Lite software. 


\section{Neutralisation assays}

509 Antibody and inhibitor neutralisation assay was performed as described previously (Montefiori,

510 2009). Briefly, virus supernatants were incubated with serial dilutions of soluble CD4 (CFAR

511 NIBSC), AMD3100 (CFAR, NIBSC), PGT151 (Falkowska et al, 2014) (gift from Laura McCoy,

$512 \mathrm{UCL}$ ) or T-20 fusion inhibitor (CFAR, NIBSC) and incubated at $37^{\circ} \mathrm{C}$ for $1 \mathrm{~h}$. The mixture was

513 added to TZM-bl cells and luciferase activity measured 48h later using Bright-Glo substrate.

514 Neutralisation was calculated as percent decrease in luciferase activity compared to virus only

515 control. $\mathrm{IC}_{50}$ values were calculated by non-linear regression analysis (sigmoid curve

516 interpolation) using Prism software (GraphPad).

\section{Statistical analysis}

519 Statistical significance was calculated where appropriate using a paired/unpaired student's $t$

520 test or one way ANOVA. Significance was assumed when $p<0.05$. All statistical analyses were calculated using Prism 9 (GraphPad Prism).

\section{Acknowledgments}

524 This work was funded by a Wellcome Trust Investigator award (108079/Z/15/Z) to C.J. SvE is supported by a grant from the National Institute of Allergy and Infectious Diseases, R01Al138625. We are grateful to Ann-Kathrin Reuschl and Maitreyi Shivkumar for helpful discussions. We also acknowledge Laura McCoy (UCL), Petra Mlcochova (DoM, Cambridge), the NIBSC Centre for AIDS Reagents and the NIH AIDS Reagent Program for reagents.

\section{Competing interests}

531 The authors declare they have no conflict of interest.

\section{References} membranes containing phosphatidylinositol-(4,5)-bisphosphate. Virology 387: 466-472 
Alfadhli A, Staubus AO, Tedbury PR, Novikova M, Freed EO \& Barklis E (2019) Analysis of HIV-1 Matrix-Envelope Cytoplasmic Tail Interactions. J Virol 93 e01079-19.

Anand SP, Grover JR, Tolbert WD, Prévost J, Richard J, Ding S, Baril S, Medjahed H, Evans DT, Pazgier M, et al (2019) Antibody-Induced Internalization of HIV-1 Env Proteins Limits Surface Expression of the Closed Conformation of Env. J Virol 93 e00293-19.

Berlioz-Torrent C, Shacklett BL, Erdtmann L, Delamarre L, Bouchaert I, Sonigo P, Dokhelar MC \& Benarous R (1999) Interactions of the Cytoplasmic Domains of Human and Simian Retroviral Transmembrane Proteins with Components of the Clathrin Adaptor Complexes Modulate Intracellular and Cell Surface Expression of Envelope Glycoproteins. J Virol 73: 1350-1361

Bhakta SJ, Shang L, Prince JL, Claiborne DT \& Hunter E (2011) Mutagenesis of tyrosine and di-leucine motifs in the HIV-1 envelope cytoplasmic domain results in a loss of Envmediated fusion and infectivity. Retrovirology 8:37.

Blattner C, Lee JH, Sliepen K, Derking R, Falkowska E, delaPeña AT, Cupo A, Julien JP, vanGils M, Lee PS, et al (2014) Structural delineation of a quaternary, cleavagedependent epitope at the gp41-gp120 interface on intact HIV-1 env trimers. Immunity 40: 669-680

Blot G, Janvier K, Le Panse S, Benarous R \& Berlioz-Torrent C (2003) Targeting of the Human Immunodeficiency Virus Type 1 Envelope to the trans-Golgi Network through Binding to TIP47 Is Required for Env Incorporation into Virions and Infectivity. $J$ Virol 77: 6931-6945

Buttler CA, Pezeshkian N, Fernandez M V., Aaron J, Norman S, Freed EO \& Van Engelenburg SB (2018) Single molecule fate of HIV-1 envelope reveals late-stage viral lattice incorporation. Nat Commun 9: 1861.

Byland R, Vance PJ, Hoxie JA \& Marsh M (2007) A conserved dileucine motif mediates clathrin and AP-2-dependent endocytosis of the HIV-1 envelope protein. Mol Biol Cell 18: 414-425

Cavrois M, De Noronha C \& Greene WC (2002) A sensitive and specific enzyme-based assay detecting HIV-1 virion fusion in primary T lymphocytes. Nat Biotechnol 20: 11511154

Checkley MA, Luttge BG \& Freed EO (2011) HIV-1 envelope glycoprotein biosynthesis, trafficking, and incorporation. J Mol Biol 410: 582-608

Chen P, Hübner W, Spinelli MA \& Chen BK (2007) Predominant Mode of Human Immunodeficiency Virus Transfer between T Cells Is Mediated by Sustained EnvDependent Neutralization-Resistant Virological Synapses. J Virol 81: 12582-12595 Cosson P (1996) Direct interaction between the envelope and matrix proteins of HIV-1. 
EMBO J 15: 5783-5788

Crooks GE, Hon G, Chandonia JM \& Brenner SE (2004) WebLogo: A sequence logo generator. Genome Res 14: 1188-1190

Deschambeault J, Lalonde J-P, Cervantes-Acosta G, Lodge R, Cohen ÉA \& Lemay G (1999) Polarized Human Immunodeficiency Virus Budding in Lymphocytes Involves a Tyrosine-Based Signal and Favors Cell-to-Cell Viral Transmission. J Virol 73: 50105017

Donzella GA, Schols D, Lin SW, Esté JA, Nagashima KA, Maddon PJ, Allaway GP, Sakmar TP, Henson G, De Clercq E, et al (1998) AMD3100, a small molecule inhibitor of HIV-1 entry via the CXCR4 co- receptor. Nat Med 4: 72-77

Durham ND \& Chen BK (2015) HIV-1 Cell-Free and Cell-to-Cell Infections Are Differentially Regulated by Distinct Determinants in the Env gp41 Cytoplasmic Tail. J Virol 89: 93249337

Van Engelenburg SB, Shtengel G, Sengupta P, Waki K, Jarnik M, Ablan SD, Freed EO, Hess HF \& Lippincott-Schwartz J (2014) Distribution of ESCRT Machinery at HIV Assembly Sites Reveals Virus Scaffolding of ESCRT Subunits. Science 343: 653-656.

Falkowska E, Le KM, Ramos A, Doores KJ, Lee JH, Blattner C, Ramirez A, Derking R, vanGils MJ, Liang $\mathrm{CH}$, et al (2014) Broadly neutralizing HIV antibodies define a glycandependent epitope on the prefusion conformation of gp41 on cleaved envelope trimers. Immunity 40: 657-668

Foley B, Korber B, Leitner T, Apetrei C, Hahn B, Mizrachi I, Mullins JI, Rambaut A \& Wolinsky S (2018) HIV Sequence Compendium 2018.

Groppelli E, Len AC, Granger LA \& Jolly C (2014) Retromer Regulates HIV-1 Envelope Glycoprotein Trafficking and Incorporation into Virions. PLoS Pathog 10 e1004518. Groppelli E, Starling S \& Jolly C (2015) Contact-Induced Mitochondrial Polarization Supports HIV-1 Virological Synapse Formation. J Virol 89: 14-24

Groves NS, Bruns MM \& van Engelenburg SB (2020) A quantitative live-cell superresolution imaging framework for measuring the mobility of single molecules at sites of virus assembly. Pathogens 9: 1-14

Hübner W, McNerney GP, Chen P, Dale BM, Gordon RE, Chuang FYS, Li XD, Asmuth DM, Huser T \& Chen BK (2009) Quantitative 3D video microscopy of HIV transfer across T cell virological synapses. Science 323: 1743-1747

Jolly C, Kashefi K, Hollinshead M \& Sattentau QJ (2004) HIV-1 Cell to Cell Transfer across an Env-induced, Actin-dependent Synapse. J Exp Med 199: 283-93.

Jolly C, Mitar I \& Sattentau QJ (2007a) Adhesion Molecule Interactions Facilitate Human Immunodeficiency Virus Type 1-Induced Virological Synapse Formation between T Cells. J Virol 81: 13916-13921 
613

614

615

616

617

618

619

620

621

622

623

624

625

626

627

628

630

631

632

633

634

635

636

637

638

639

640

641

642

643

644

645

646

647

648

649

Jolly C, Mitar I \& Sattentau QJ (2007b) Requirement for an Intact T-Cell Actin and Tubulin Cytoskeleton for Efficient Assembly and Spread of Human Immunodeficiency Virus Type 1. J Virol 81: 5547-5560.

Kalia V, Sarkar S, Gupta P \& Montelaro RC (2003) Rational Site-Directed Mutations of the LLP-1 and LLP-2 Lentivirus Lytic Peptide Domains in the Intracytoplasmic Tail of Human Immunodeficiency Virus Type $1 \mathrm{gp} 41$ Indicate Common Functions in Cell-Cell Fusion but Distinct Roles in Virion Envelope Incorpora. J Virol 77: 3634-3646

Kirschman J, Qi M, Ding L, Hammonds J, Dienger-Stambaugh K, Wang J-J, Lapierre LA, Goldenring JR \& Spearman P (2017) HIV-1 Envelope Glycoprotein Trafficking through the Endosomal Recycling Compartment Is Required for Particle Incorporation. J Virol 92: e01893-17.

Kubo Y, Tominaga C, Yoshii H, Kamiyama H, Mitani C, Amanuma H \& Yamamoto N (2007) Characterization of $\mathrm{R}$ peptide of murine leukemia virus envelope glycoproteins in syncytium formation and entry. Arch Virol 152: 2169-2182

Lambelé M, Labrosse B, Roch E, Moreau A, Verrier B, Barin F, Roingeard P, Mammano F \& Brand D (2007) Impact of Natural Polymorphism within the gp41 Cytoplasmic Tail of Human Immunodeficiency Virus Type 1 on the Intracellular Distribution of Envelope Glycoproteins and Viral Assembly. J Virol 81:125-40.

Lodge R, Göttlinger H, Gabuzda D, Cohen EA \& Lemay G (1994) The intracytoplasmic domain of gp41 mediates polarized budding of human immunodeficiency virus type 1 in MDCK cells. J Virol 68: 4857-4861

Lu L, Zhu Y, Huang J, Chen X, Yang H, Jiang S \& Chen YH (2008) Surface exposure of the HIV-1 Env cytoplasmic tail LLP2 domain during the membrane fusion process: Interaction with gp41 fusion core. J Biol Chem 283: 16723-16731

Mesner D, Hotter D, Kirchhoff F \& Jolly C (2020) Loss of Nef-mediated CD3 down-regulation in the HIV-1 lineage increases viral infectivity and spread. Proc Natl Acad Sci U S A 117: 7382-7391.

Monit C, Goldstein RA \& Towers GJ (2019) ChromaClade: Combined visualisation of phylogenetic and sequence data. BMC Evol Biol 19: 186.

Montefiori DC (2009) Measuring HIV neutralization in a luciferase reporter gene assay. Methods Mol Biol 485: 395-405

Morita E, Sandrin V, McCullough J, Katsuyama A, Baci Hamilton I \& Sundquist WI (2011) ESCRT-III protein requirements for HIV-1 budding. Cell Host Microbe 9: 235-242 Muñoz-Barroso I, Salzwedel K, Hunter E \& Blumenthal R (1999) Role of the MembraneProximal Domain in the Initial Stages of Human Immunodeficiency Virus Type 1 Envelope Glycoprotein-Mediated Membrane Fusion. J Virol 73: 6089-6092 Murakami T \& Freed EO (2000) The long cytoplasmic tail of gp41 is required in a cell type- 
650

dependent manner for HIV-1 envelope glycoprotein incorporation into virions. Proc Natl Acad Sci U S A 97: 343-8.

Murooka TT, Deruaz M, Marangoni F, Vrbanac VD, Seung E, Von Andrian UH, Tager AM, Luster AD \& Mempel TR (2012) HIV-infected T cells are migratory vehicles for viral dissemination. Nature 490: 283-289

Murphy RE \& Saad JS (2020) The interplay between HIV-1 gag binding to the plasma membrane and env incorporation. Viruses 12

Murphy RE, Samal AB, Vlach J \& Saad JS (2017) Solution Structure and Membrane Interaction of the Cytoplasmic Tail of HIV-1 gp41 Protein. Structure 25: 1708-1718.e5

Ohno H, Aguilar RC, Fournier MC, Hennecke S, Cosson P \& Bonifacino JS (1997) Interaction of endocytic signals from the HIV-1 envelope glycoprotein complex with members of the adaptor medium chain family. Virology 238: 305-315

Ono A, Ablan SD, Lockett SJ, Nagashima K \& Freed EO (2004) Phosphatidylinositol $(4,5)$ bisphosphate regulates HIV-1 Gag targeting to the plasma membrane. Proc Natl Acad Sci U S A 101:14889-94.

Ono A, Huang M \& Freed EO (1997) Characterization of human immunodeficiency virus type 1 matrix revertants: effects on virus assembly, Gag processing, and Env incorporation into virions. J Virol 71:4409-18.

Pezeshkian N, Groves NS \& van Engelenburg SB (2019) Single-molecule imaging of HIV-1 envelope glycoprotein dynamics and Gag lattice association exposes determinants responsible for virus incorporation. Proc Natl Acad Sci U S A 116: 25269-25277

Piai A, Fu Q, Cai Y, Ghantous F, Xiao T, Shaik MM, Peng H, Rits-Volloch S, Chen W, Seaman MS, et al (2020) Structural basis of transmembrane coupling of the HIV-1 envelope glycoprotein. Nat Commun 11:2317.

Pizzato M, Erlwein O, Bonsall D, Kaye S, Muir D \& McClure MO (2009) A one-step SYBR Green I-based product-enhanced reverse transcriptase assay for the quantitation of retroviruses in cell culture supernatants. $J$ Virol Methods 156:1-7.

Postler TS \& Desrosiers RC (2013) The Tale of the Long Tail: the Cytoplasmic Domain of HIV-1 gp41. J Virol 87: 2-15

Prescher J, Baumgärtel V, Ivanchenko S, Torrano AA, Bräuchle C, Müller B \& Lamb DC (2015) Super-Resolution Imaging of ESCRT-Proteins at HIV-1 Assembly Sites. PLoS Pathog 10: e1004677.

Qi M, Williams JA, Chu H, Chen X, Wang JJ, Ding L, Akhirome E, Wen X, Lapierre LA, Goldenring JR, et al (2013) Rab11-FIP1C and Rab14 Direct Plasma Membrane Sorting and Particle Incorporation of the HIV-1 Envelope Glycoprotein Complex. PLoS Pathog 9: e1003278.

Rudnicka D, Feldmann J, Porrot F, Wietgrefe S, Guadagnini S, Prévost M-C, Estaquier J, 
687

688

689

690

691

692

693

694

695

696

697

698

699

700

701

702

703

704

705

706

707

708

709

710

711

712

713

714

715

716

717

718

719

720

721

722

723

Haase AT, Sol-Foulon N \& Schwartz O (2009) Simultaneous Cell-to-Cell Transmission of Human Immunodeficiency Virus to Multiple Targets through Polysynapses. $J$ Virol 83: 6234-6246

Rusert P, Kouyos RD, Kadelka C, Ebner H, Schanz M, Huber M, Braun DL, Hozé N, Scherrer A, Magnus C, et al (2016) Determinants of HIV-1 broadly neutralizing antibody induction. Nat Med 22: 1260-1267

Salzwedel K, West JT \& Hunter E (1999) A Conserved Tryptophan-Rich Motif in the Membrane-Proximal Region of the Human Immunodeficiency Virus Type $1 \mathrm{gp} 41$ Ectodomain Is Important for Env-Mediated Fusion and Virus Infectivity. J Virol 73: 2469-2480

Sattentau Q (2008) Avoiding the void: Cell-to-cell spread of human viruses. Nat Rev Microbiol 6:815-26.

Schindelin J, Arganda-Carreras I, Frise E, Kaynig V, Longair M, Pietzsch T, Preibisch S, Rueden C, Saalfeld S, Schmid B, et al (2012) Fiji: An open-source platform for biological-image analysis. Nat Methods 9: 676-682

Sourisseau M, Sol-Foulon N, Porrot F, Blanchet F \& Schwartz O (2007) Inefficient Human Immunodeficiency Virus Replication in Mobile Lymphocytes. J Virol 81: 1000-1012

Stano A, Leaman DP, Kim AS, Zhang L, Autin L, Ingale J, Gift SK, Truong J, Wyatt RT, Olson AJ, et al (2017) Dense Array of Spikes on HIV-1 Virion Particles. J Virol 91 : e00415-17

Starling S., \& Jolly C. (2016) LFA-1 engagement triggers T cell polarization at the HIV-1 virological synapse. J Virol 21: 9841-54.

Stuchell MD, Garrus JE, Müller B, Stray KM, Ghaffarian S, McKinnon R, Kräusslich HG, Morham SG \& Sundquist WI (2004) The human endosomal sorting complex required for transport (ESCRT-I) and its role in HIV-1 budding. J Biol Chem 279: 36059-36071 Tedbury PR, Ablan SD \& Freed EO (2013) Global Rescue of Defects in HIV-1 Envelope Glycoprotein Incorporation: Implications for Matrix Structure. PLoS Pathog 9: e1003739.

Tremblay M, Rooke R, Geleziunas R, Wainberg MA, Sullivan AK, Tsoukas C, Gilmore N \& Shematek G (1989) New CD4( +) cell line susceptible to infection by HIV-1. J Med Virol 28: 243-249

Wang L, Sandmeyer A, Hübner W, Li H, Huser T \& Chen BK (2020) Recruitment of Env to the HIV-1 T cell virological synapse by targeted and 2 sustained Env recycling. bioRxiv https://doi.org/10.1101/2020.12.08.417188

Wyss S, Berlioz-Torrent C, Boge M, Blot G, Höning S, Benarous R \& Thali M (2001) The Highly Conserved C-Terminal Dileucine Motif in the Cytosolic Domain of the Human Immunodeficiency Virus Type 1 Envelope Glycoprotein Is Critical for Its Association 
with the AP-1 Clathrin Adapter. J Virol 75: 2982-2992

Wyss S, Dimitrov AS, Baribaud F, Edwards TG, Blumenthal R \& Hoxie JA (2005) Regulation of Human Immunodeficiency Virus Type 1 Envelope Glycoprotein Fusion by a Membrane-Interactive Domain in the gp41 Cytoplasmic Tail. J Virol 79: 12231-12241

\section{Figure Legends}

Figure 1. EnvCT W757A does not polarise to viral synapses and leads to a defect in cell-

cell spread. (A) Schematic showing the HIV-1 NL4.3 EnvCT sequence and location of W757 in the LLP2 alpha helix. Logo plots (generated using WebLogo3) indicate the probability of 5916 HIV-1 Env sequences analysed in the LANL database containing a W residue at the indicated positions. (B) Synapse formation between Jurkat cells infected with WT NL4.3 (left panel) or the W757A mutant (right panel) and uninfected target cells. NL4-3 virus pseudotyped with VSV-G to normalise viral entry was used to infect donor Jurkat cells. Representative image is an $x y$ slice through the middle of a cell-cell contact between an infected donor cell (Env, green; Gag, red) and a target cell stained with a cell trace dye (blue). (C) Percent of contacts that exhibited synapse formation (defined by polarisation of both Env and Gag to the contact site). (D) Quantification of the percentage of infected donor cells (Gag+) in contact with target cells (blue). Data show the mean and SEM from $n=54-69$ infected donor cells compared using a two-tailed paired t test (ns, not significant; $\left.{ }^{* \star *} \mathrm{p}<0.001\right)$. (E) Signal intensity enrichment at the synapse, relative to a synapse distal site was determined from 20 images using ImageJ analysis. (F) Infected donor cells were mixed in a 1:1 ratio with target cells

746 (stained with cell trace far red) and viral spread was measured by intracellular Gag staining in 747 target cells by flow cytometry. Data show the percent of Gag+ target cells over time. Jurkat 748 cells (left) and primary CD4+ cells (right). Data show the mean and SEM from four 749 independent Jurkat cells experiments analysed using multiple paired t tests (ns, not significant;

$\left.750{ }^{* \star *} p<0.001\right)$. For primary $\mathrm{CD}^{+} \mathrm{T}$ cells a representative donor from three unique donors is shown. 

sites. CEM-A adherent T cells were transduced with WT and W757A mutant Envelope virus and imaged after 48 hours. (A) Super-resolution reconstructions of Gag and lattice proximal/distal single-molecule localisations of Env are demonstrated (Scale bars $=200 \mathrm{~nm}$. Arrows highlight highly confined single molecule localisations of Env). Proximal and distal single-molecule localisations show the confined and freely-diffusing nature of single Env trimers, respectively. (B) Wild-type and mutant Env trimers do not differ in mobility when lattice proximal (top panels) (WT: $D_{\text {apparent }}=0.038 \pm 0.06 \mu \mathrm{m}^{2} \mathrm{~s}^{-1}, S_{M S S}=0.03 \pm 0.13$; W757A: $\left.D_{\text {apparent }}=0.033 \pm 0.03 \mu^{2} s^{-1}, S_{M S S}=0.045 \pm 0.16\right)$, whereas lattice-distal diffusion differs significantly between viruses (bottom panels) (WT: $D_{\text {apparent }}=0.07 \pm 0.17 \mu^{2} s^{-1}$, $S_{M S S}=0.12 \pm 0.21 ;$ W757A: $\left.D_{\text {apparent }}=0.05 \pm 0.10 \mu^{2} s^{-1}, S_{M S S}=0.13 \pm 0.22\right)$. Statistical significance was determined by unpaired two-sample t-test. (Proximal: $P_{D}=0.0693, P_{S_{M S S}}=$ 0.3743; Distal: $\left.P_{D}<0.0001, P_{S_{M S S}}=0.0017\right)$. Error represents S.D.

Figure 3. EnvCT W757 is required for HIV-1 budding and infectivity. (A) Virus release from infected Jurkat and primary $\mathrm{CD}^{+} \mathrm{T}$ cells at $48 \mathrm{hpi}$ measured by quantifying $\mathrm{RT}$ activity in viral supernatants by SG-PERT qPCR. (B) Infectivity of virus particles released 48hpi was measured by titrating virus on HeLa TZM-bl reporter cells and RLU normalised per RT unit to calculate relative particle infectivity. (C) Western blot of cell lysate and purified virus from infected primary $\mathrm{CD}^{+} \mathrm{T}$ cells at 48hpi probed with anti-Env (gp120/gp160) and anti-Gag (p55/p24). (D) Quantification of Env (gp120) incorporation into virions normalised to Gag (p24) density, produced in Jurkat and primary $\mathrm{CD} 4^{+} \mathrm{T}$ cells. Data show the mean and SEM from at least three independent experiments compared using a two-tailed paired t test (ns, not significant; $\left.{ }^{* * *} p<0.0001\right)$.

EnvCT. (A) Intracellular staining of Env (green) and Gag (red) in Jurkat cells infected with WT 
and W757A at 48hpi. (B) Western blot of infected Jurkat cell lysates harvested 48hpi and probed with anti-gp120, anti-gp41, and anti-Gag p55/p24. Quantification of Env processing (gp120/gp160). Data show the mean and SEM from at least three independent experiments compared using a two-tailed paired t test (ns, not significant). (C) Flow cytometry analysis of cell surface Env levels on cells infected with WT (black) and W757A (grey) virus measured using bNAb PGT151. Uninfected control is shown in light grey. (D) Time course of endocytosed Env recycling back to the plasma membrane measured by flow cytometry. Env was stained with either $2 \mathrm{G} 12$ or PGT151. Envelope recycling was measured by staining with envelope antibody and a PE-secondary on ice followed by Cy5 at $37^{\circ} \mathrm{C}$. Recycling is shown as the MFI of $\mathrm{PE}^{+} \mathrm{CY}^{+}$signal on the surface over time. Data show the mean and SEM from three independent experiments.

791

Figure 5. W757 A virus shows a defect in viral fusion (A) Neutralisation of WT and W757A virus (produced from Jurkat T cells) by PGT151 bNAb, sCD4, AMD3100 and T20. IC 50 values calculated by non-linear regression analysis of the neutralisation curves. ND denotes not determined. (B) W757A Env is defective in viral fusion compared to WT virus as measured by the BlaM-Vpr fusion assay. Data are the mean and SEM compared using an unpaired test $\left({ }^{* *} \mathrm{p}<0.001\right)$. Mock refers to co-culture between uninfected T cells and target $\mathrm{T}$ cells.

Figure 6. Conservation of W757 across HIV and SIV lineages. (A) Amino acid alignment

800 of the LLP2 domain of the EnvCT from different HIV and SIV consensus sequences. See also

801 Supplementary Figure S4. (B-D) Viral budding, infectivity, and cell-cell spread can be rescued 802 by substitution at position 757 with either tyrosine $\mathrm{Y}$, or phenylalanine, F. Data are the mean and SEM compared using a two-tailed paired t test (ns, not significant; $\left.{ }^{* * *} p<0.001\right)$.

\section{Supplemental Information}


807 Supplementary Figure 1. EnvCT W757A is defective in $\mathbf{T}$ cell $-\mathbf{T}$ cell spread.

808 Representative flow cytometry data of cell-cell spread between Jurkat T cells (A) and primary

$809 \mathrm{CD}^{+} \mathrm{T}$ cells (B). The percentage of cells in each quadrant is indicted.

811 Supplementary Figure 2. EnvCT W790A virus does not perturb HIV-1 Env function and

812 viral cell-cell spread. (A) Alanine substitution of the tryptophan at HIV-1 envelope position

813790 does not affect the ability of infected Jurkat cells to form conjugates with uninfected target

814 cells. (B-C) W790A infected cells also form synapses to the same extent at WT HIV-1 and

815 result in no defect in both intracellular and surface localization of Env. (D) Quantification of

816 Env (gp120) incorporation into virions produced in Jurkat cells, normalised to Gag (p24)

817 density. (E) Cell surface W790A Envelope (grey) measured by flow cytometry is equal to WT

818 (black) levels. (F-G) Budding of virons from infected cells, and the relative infectivity of virions

819 is equivalent to WT. (H) Viral transfer via cell-cell spread is the same with WT Env and W790A

820 Env, but is significantly defective in W757A Env infection.

821

Supplementary Figure 3. Compensatory Matrix mutant (Q62R) is unable to alleviate the W757A phenotype. MA mutation Q62R does not rescue the defect in W757A budding (A) or infectivity (B). Data are the mean and SEM compared using a one way ANOVA (ns, not significant; $\left.{ }^{* \star} p<0.01 ;{ }^{* \star * *} p<0.0001\right)$.

Supplementary Figure 4. Conservation of W757 among HIV-1 M group, SIVcpz, and SIVgor envelope glycoprotein. Phylogenetic analysis of the EnvCT showing amino acid residues found at position 757 (based on 194 consensus envelope sequences from 830 http://www.hiv.lanl.gov/ ) performed using Chromaclade. Tryptophan (W) - dark blue. Tyrosine 831 (Y) - light blue. Isoleucine (I) - purple. Leucine (L) - turquoise. 
835 plasma membrane. Trytophan (red) and Alanine (blue) are highlighted in (C). The aromatic

836 side chain of W757 (indicated by the yellow arrow) is predicted to be buried into the

837 membrane, maintaining the EnvCT baseplate conformation, whereas alanine substitution at

838 this position allows for more conformational flexibility in the quaternary structure of the EnvCT

839 baseplate, disrupting the TMD and perhaps MPER/gp120 conformations.

840 
A
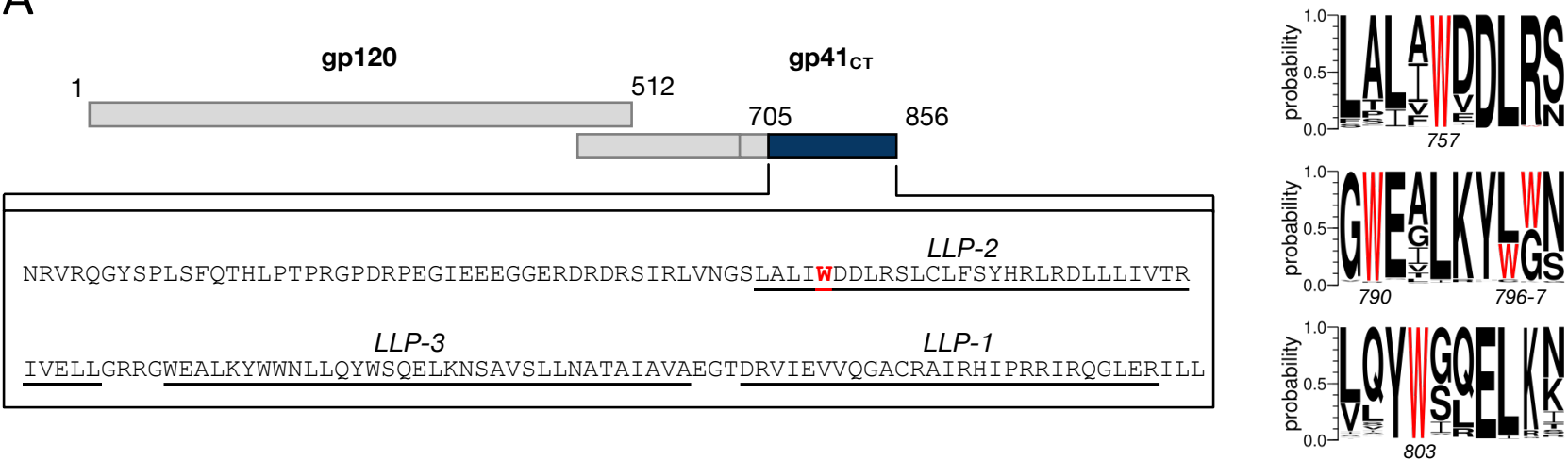

B

C

D

$E$
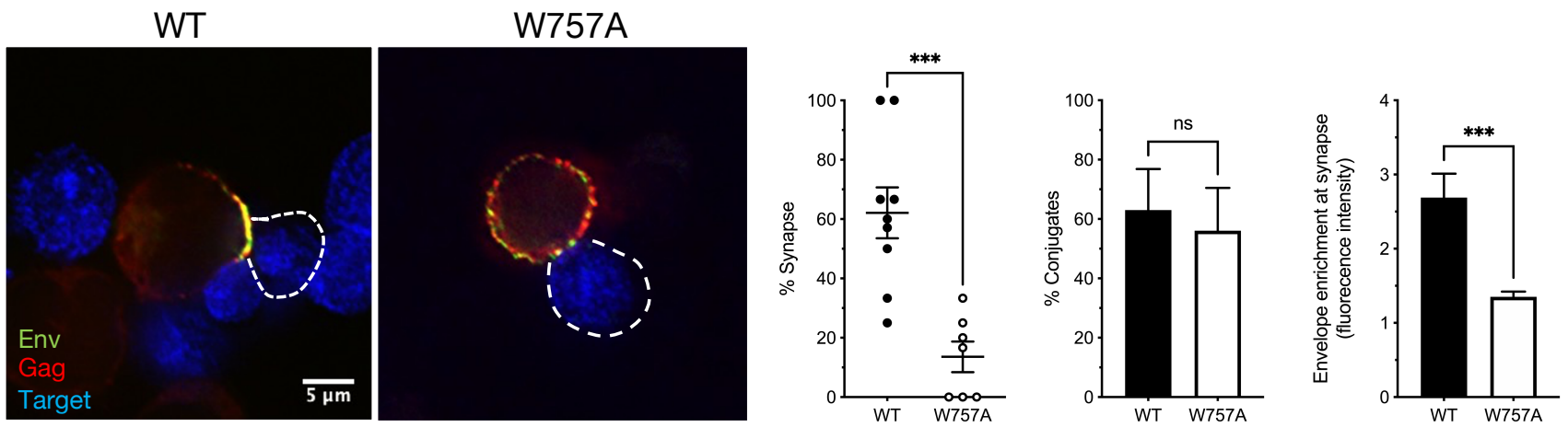

$\mathrm{F}$

Jurkat : Jurkat

Primary : Primary
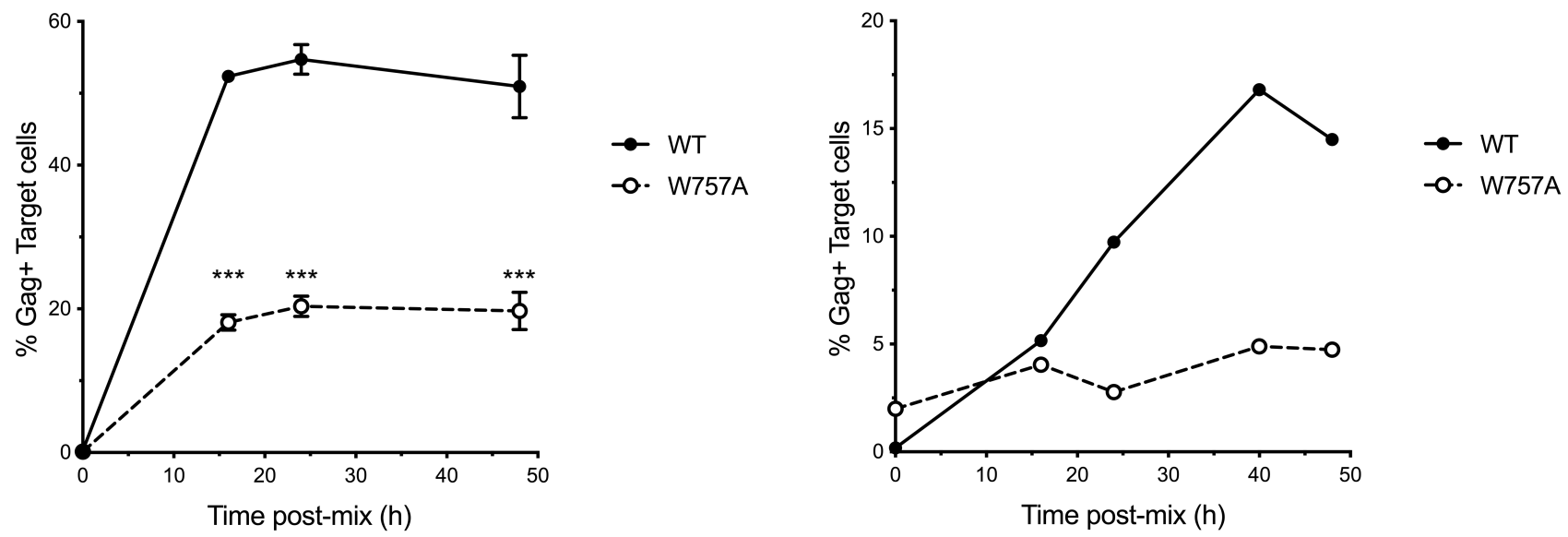

Figure 1. EnvCT W757A does not polarise to viral synapses and leads to a defect in cell-cell spread. (A) Schematic showing the HIV-1 NL4.3 EnvCT sequence and location of W757 in the LLP2 alpha helix. Logo plots (generated using WebLogo3) indicate the probability of 5916 HIV-1 Env sequences analysed in the LANL database containing a W residue at the indicated positions. (B) Synapse formation between Jurkat cells infected with WT NL4.3 (left panel) or the W757A mutant (right panel) and uninfected target cells. NL4-3 virus pseudotyped with VSVg to normalise viral entry was used to infect donor Jurkat cells. Representative image is an $x y$ slice through the middle of a cell-cell contact between an infected donor cell (Env, green; Gag, red) and a target cell stained with a cell trace dye (blue). (C) Percent of contacts that exhibited synapse formation (defined by polarisation of both Env and Gag to the contact site). (D) Quantification of the percentage of infected donor cells (Gag+) in contact with target cells (blue). Data show the mean and SEM from n=54-69 infected donor cells compared using a two-tailed paired t test (ns, not significant; $\left.{ }^{* * *} p<0.001\right)$. (E) Signal intensity enrichment at the synapse, relative to a synapse distal site was determined from 20 images using ImageJ analysis. (F) Infected donor cells were mixed in a 1:1 ratio with target cells (stained with cell trace far red) and viral spread was measured by intracellular Gag staining in target cells by flow cytometry. Data show the percent of Gag+ target cells over time. Jurkat cells (left) and primary CD4+ cells (right). Data show the mean and SEM from four independent Jurkat cells experiments analysed using multiple paired t tests (ns, not significant; ${ }^{* * *} p<0.001$ ). For primary $\mathrm{CD}^{+} \mathrm{T}$ cells a representative donor from three unique donors is shown. 
A

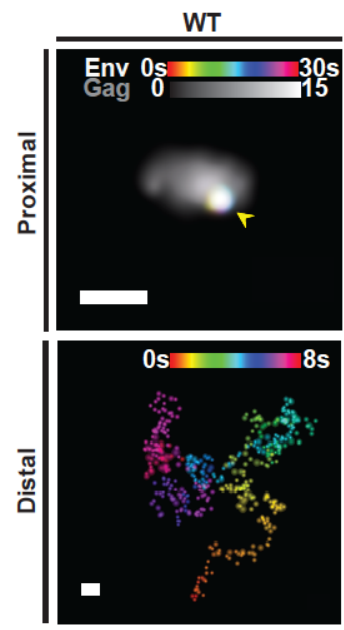

B
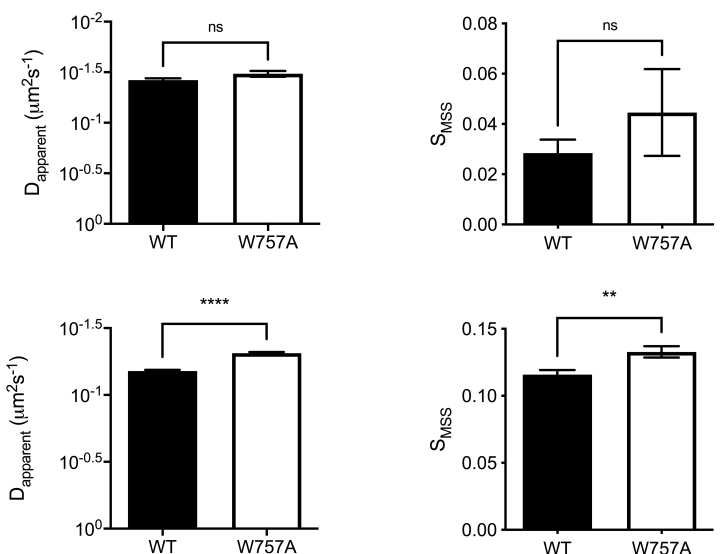

Figure 2. W757A EnvCT does not display a Gag lattice trapping defect at virus assembly sites. CEM-A adherent $T$ cells were transduced with WT and W757A mutant Envelope virus and imaged after 48 hours. (A) Super-resolution reconstructions of Gag and lattice proximal/distal single-molecule localisations of Env are demonstrated (Scale bars = $200 \mathrm{~nm}$. Arrows highlight highly confined single molecule localisations of Env). Proximal and distal single-molecule localisations show the confined and freely-diffusing nature of single Env trimers, respectively. (B). Wild-type and mutant Env trimers do not differ in mobility when lattice proximal (top panels) (WT: $D_{\text {apparent }}=0.038 \mu \mathrm{m}^{2} \mathrm{~s}^{-1}, S_{M S S}=0.01$; W757A: $D_{\text {apparent }}=0.033 \mu^{2} s^{-1}, S_{M S S}=0.045$ ), whereas lattice-distal diffusion differs significantly between viruses (bottom panels) (WT: $D_{\text {apparent }}=0.066 \mu^{2} s^{-1}, S_{M S S}=0.116 ;$ W757A: $D_{\text {apparent }}=0.049 \mu^{2} s^{-1}, S_{M S S}=0.133$ ). Statistical significance was determined by unpaired two-sample t-test. (Proximal: $P_{D}=9.22 \times 10^{-24}, P_{S_{M S S}}=0.29$; Distal: $P_{D}=0.19$, $\left.P_{S_{M S S}}=0.0016\right)$. 
Jurkat

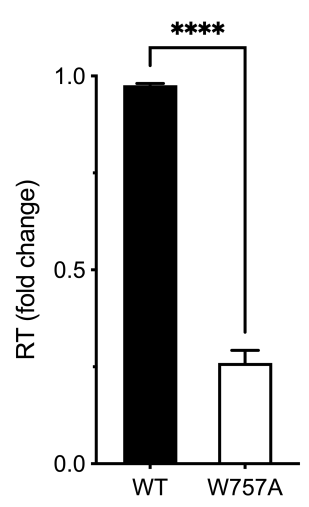

Primary CD4+ $\mathrm{T}$ cells

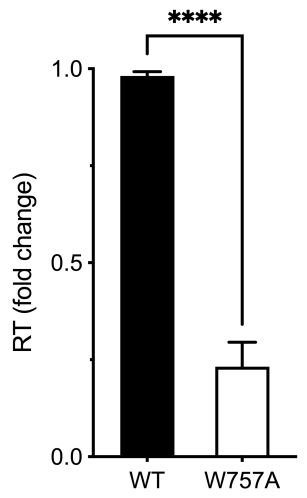

Jurkat

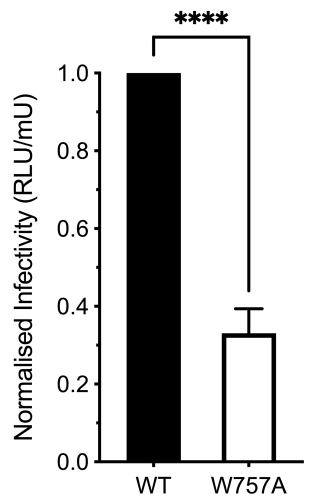

Primary CD4 ${ }^{+} \mathrm{T}$ cells

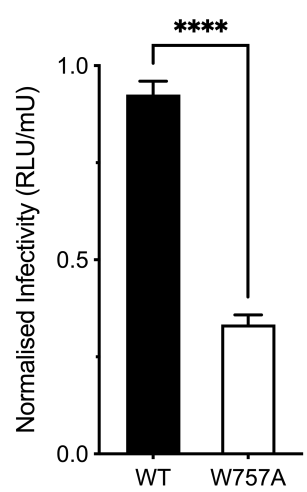

C

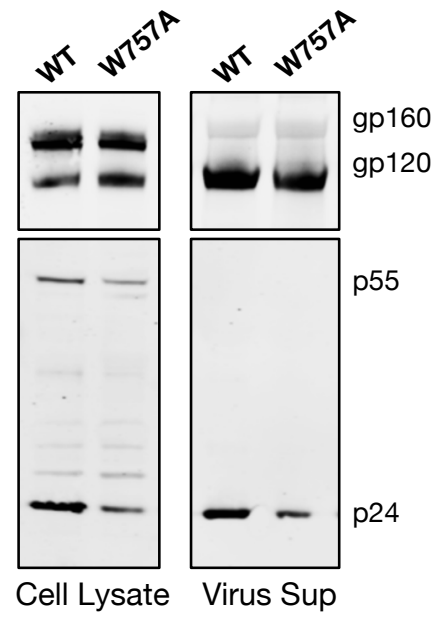

D

\section{Env Incorporation}

Jurkat

Primary CD4 ${ }^{+} \mathrm{T}$ cells
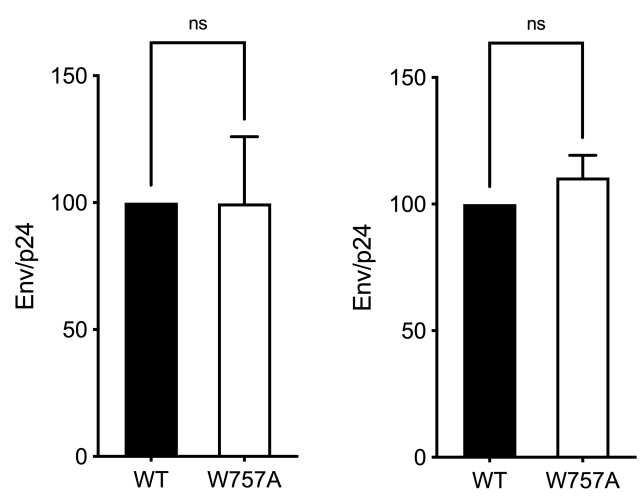

Figure 3. EnvCT W757 is required for HIV-1 budding and infectivity. (A) Virus release from infected Jurkat and primary $\mathrm{CD}^{+} \mathrm{T}$ cells at $48 \mathrm{hpi}$ measured by quantifying RT activity in viral supernatants by SG-PERT qPCR. (B) Infectivity of virus particles released 48hpi was measured by titrating virus on HeLa TZM-bl reporter cells and RLU normalised per RT unit to calculate relative particle infectivity. (C) Western blot of cell lysate and purified virus from infected primary CD4 ${ }^{+} \mathrm{T}$ cells at 48hpi probed with anti-Env (gp120/gp160) and anti-Gag (p55/p24). (D) Quantification of Env (gp120) incorporation into virions normalised to Gag (p24) density, produced in Jurkat and primary CD4 ${ }^{+} \mathrm{T}$ cells. Data show the mean and SEM from at least three independent experiments compared using a two-tailed paired t test (ns, not significant; ${ }^{* * * *} p<0.0001$ ). 
A

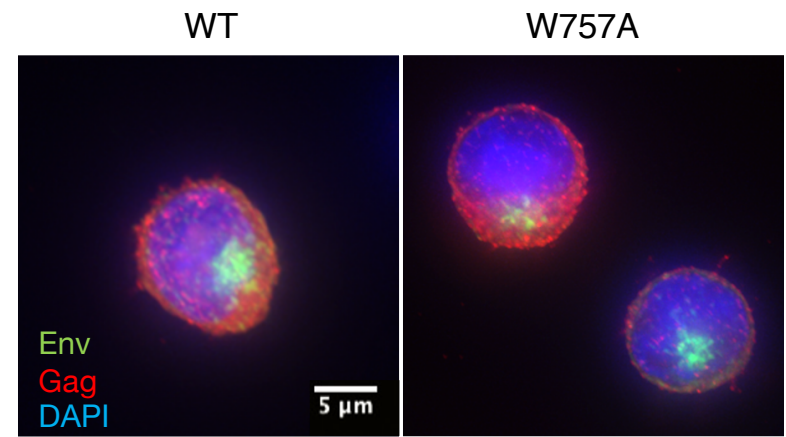

B

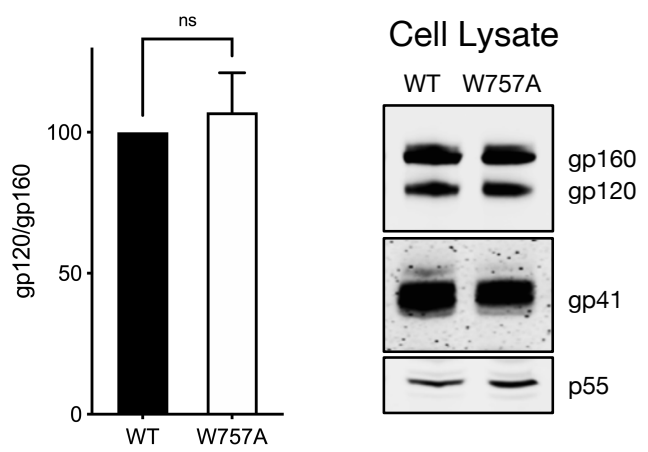

C

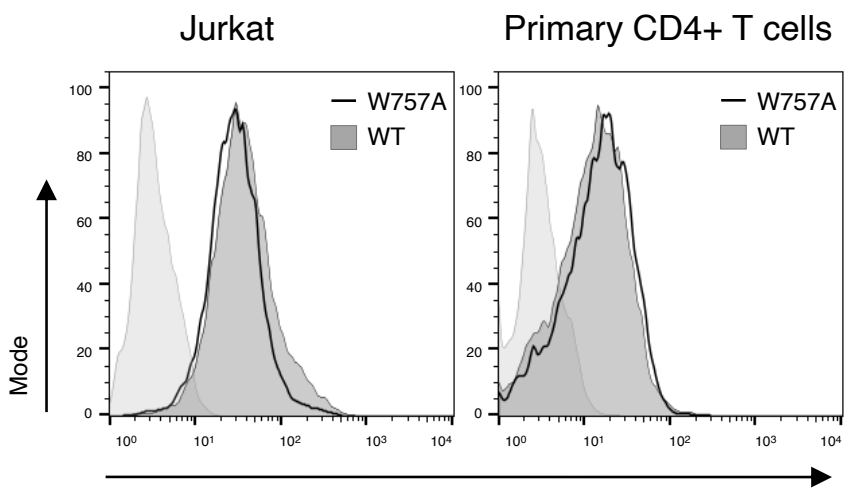

Env MFI (PGT151)
D

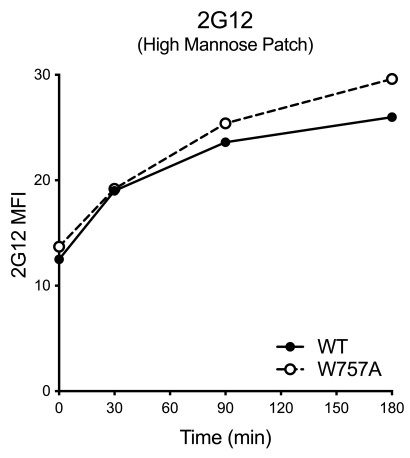

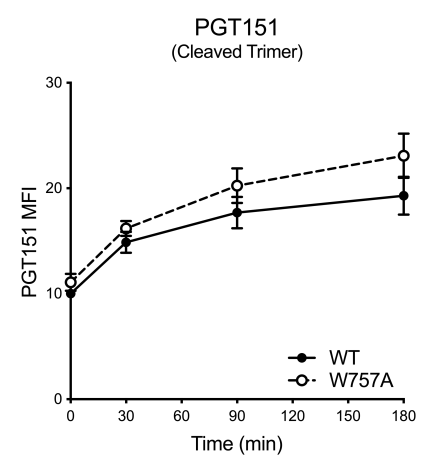

Figure 4. Synthesis, processing, and recycling of EnvCT W757A is equivalent to WT EnvCT. (A) Intracellular staining of Env (green) and Gag (red) in Jurkat cells infected with WT and W757A at 48hpi. (B) Western blot of infected Jurkat cell lysates harvested 48hpi and probed with anti-gp120, anti-gp41, and anti-Gag p55/p24. Quantification of Env processing (gp120/gp160). Data show the mean and SEM from at least three independent experiments compared using a two-tailed paired t test (ns, not significant). (C) Flow cytometry analysis of cell surface Env levels on cells infected with WT (black) and W757A (grey) virus measured using bNAb PGT151. Uninfected control is shown in light grey. (D) Time course of endocytosed Env recycling back to the plasma membrane measured by flow cytometry. Env was stained with either $2 \mathrm{G} 12$ or PGT151. Envelope recycling was measured by staining with envelope antibody and a PE-secondary on ice followed by Cy5 at $37^{\circ} \mathrm{C}$. Recycling is shown as the MFI of $\mathrm{PE}^{+} \mathrm{CY}^{+}$signal on the surface over time. Data show the mean and SEM from three independent experiments. 


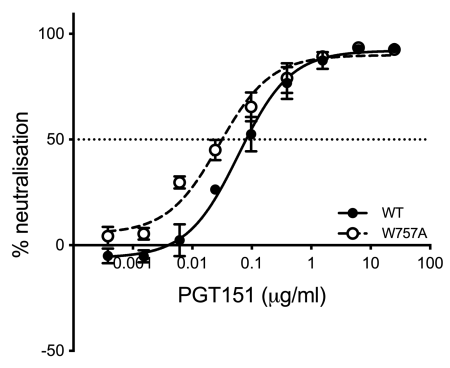

\begin{tabular}{|c|c|c|}
\hline & WT & W757A \\
\hline IC50 $(\mu \mathrm{g} / \mathrm{ml})$ & 0.06 & 0.03 \\
\hline
\end{tabular}

AMD3100

(Jurkat - TZMb)

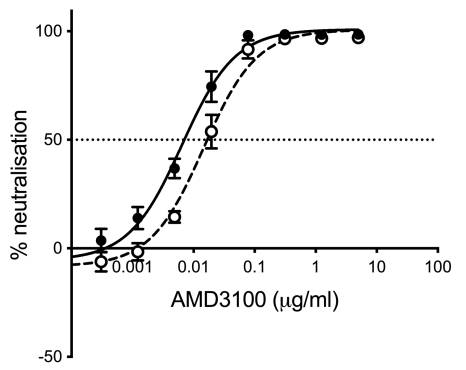

\begin{tabular}{|c|c|c|}
\hline & WT & W757A \\
\hline IC50 $(\mu \mathrm{g} / \mathrm{ml})$ & 0.006 & 0.015 \\
\hline
\end{tabular}

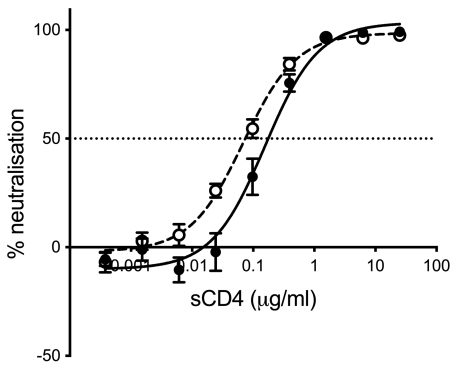

\begin{tabular}{|c|c|c|}
\hline & WT & W757A \\
\hline IC50 $(\mu \mathrm{g} / \mathrm{ml})$ & 0.16 & 0.07 \\
\hline
\end{tabular}

Enfuvirtide (T20) (Jurkat - TZMbl)

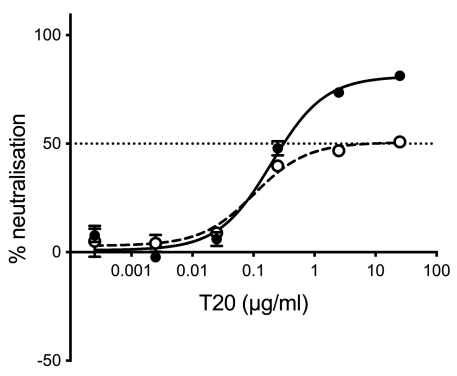

\begin{tabular}{|c|c|c|}
\hline & WT & W757A \\
\hline IC50 $(\mu \mathrm{g} / \mathrm{ml})$ & 0.198 & ND \\
\hline
\end{tabular}

B
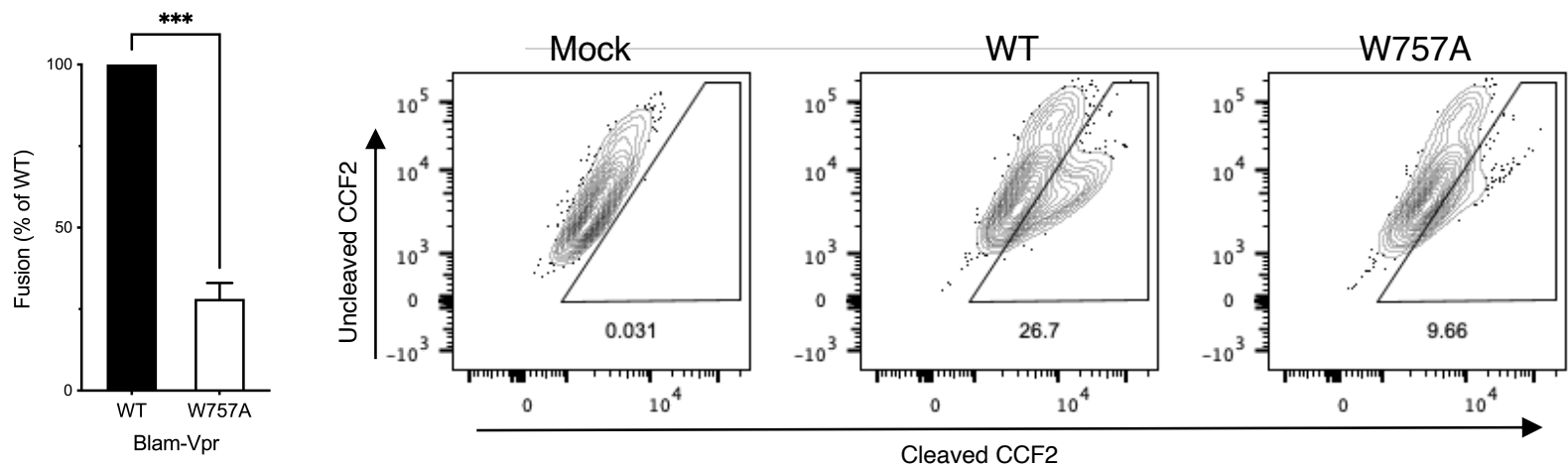

Figure 5. W757A virus shows a defect in viral fusion. (A) Neutralisation of WT and W757A virus (produced from Jurkat T cells) by PGT151 bNAb, sCD4, AMD3100 and T20. IC 50 values calculated by non-linear regression analysis of the neutralisation curves. ND denotes not determined. (B) W757A Env is defective in viral fusion compared to WT virus as measured by the BlaM-Vpr fusion assay. Data are the mean and SEM compared using an unpaired $t$ test $\left({ }^{* * *} p<\right.$ 0.001). Mock refers to co-culture between uninfected $T$ cells and target $T$ cells. 
A

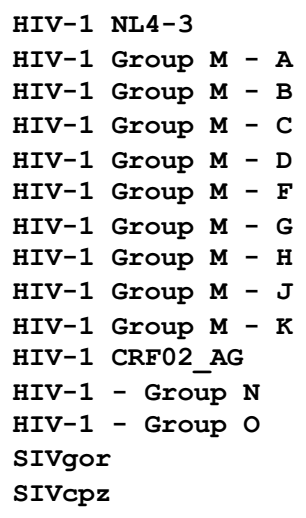

RDRDRSIRLVNGSLALIWDDLRSLCLFSYHRLRDLLLI 797 QDRGRSIRLVSGFLALAWDDLRNLCLFSYHRLRDFILI 797 RDRDRS IRLVNGSLALIWDDLRSLCLFSYHRLRDLLLI 797 QGRDRS IRLVNGFLAIFWDDLRSLCLFSYHRLRDLILI 797 QGRDRS IRLVNGFSALIWDDLRNLCLFSYHRLRDLILI 797 QGKDRSVRLVTGFLALAWDDLRNLCLFSYRHLRDFILI 797 QDRDRSTRLVSGFLALAWDDLRSLCLFSYHRLRDLVLI 797 QDRDRSVRLVNGFLPVVWDDLRSLSLFSYRLLRDLLLI 797 QGRTRSIRLVSGFLALAWDDLRSLCLFSYHRLRDFVLI 797 QDKDRSVRLVSGFLALAWDDLRNLCLFSYRHLRDLVLI 797 QGRDRSVRLVSGFLTLAWDDLRSLCLFSYHRLRDFILI 797 QDRGRSVRLVSGFLALIWEDLRNLLIFLYHRLADSLLI 798 EGRPRWIPSPQGFLPLLYTDLRTIILWTYHLLSNLASG 797 ADSDRSIRLLAGFLPLIWDDLRTLVVWIYRTLVILLSG 805 QGRGRSVRLVTGCLPLIWDDLRSLLIWFYQTLSNSACV 797
B

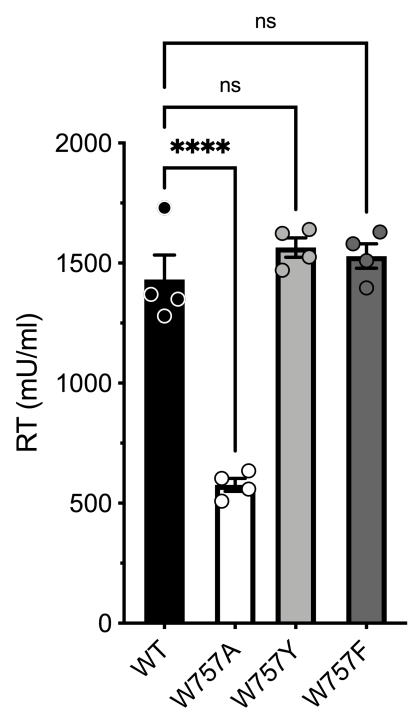

NL4.3 Env
C

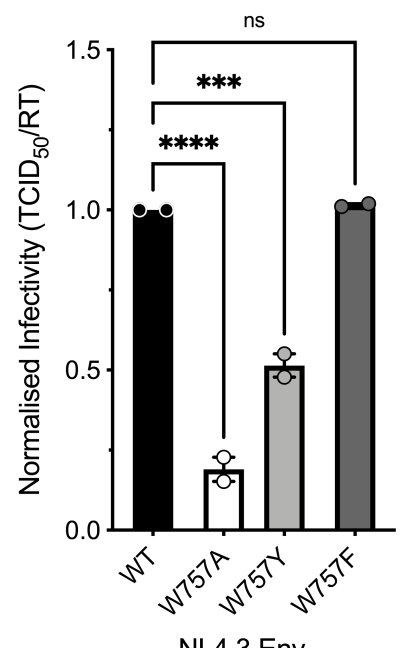

D

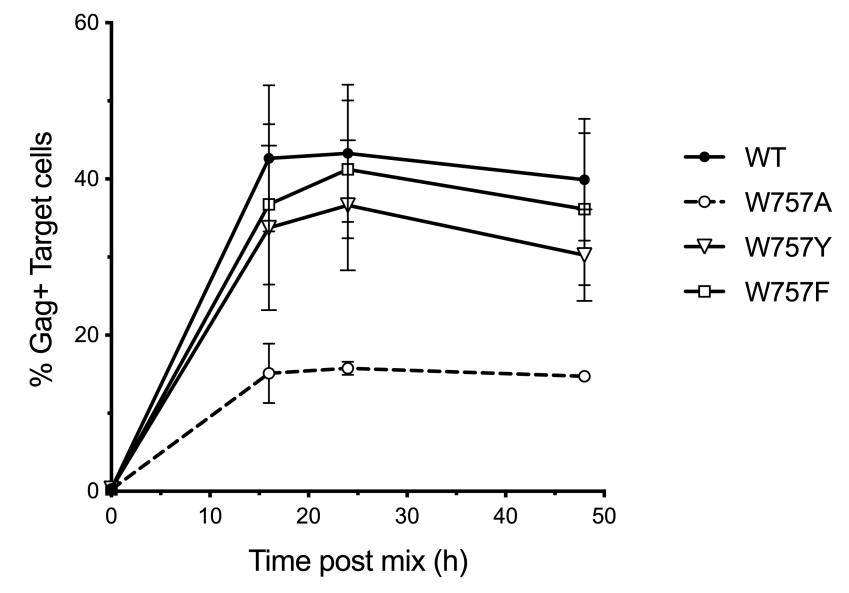

Figure 6. Conservation of W757 across HIV and SIV lineages. (A) Amino acid alignment of the LLP2 domain of the EnvCT from different HIV and SIV consensus sequences. See also Supplementary Figure S4. (B-D) Viral budding, infectivity, and cell-cell spread can be rescued by substitution at position 757 with either tyrosine $\mathrm{Y}$, or phenylalanine, $\mathrm{F}$. Data are the mean and SEM compared using a two-tailed paired $t$ test (ns, not significant; $\left.{ }^{* * *} p<0.001\right)$. 
A

WT

(Jurkat:Jurkat)
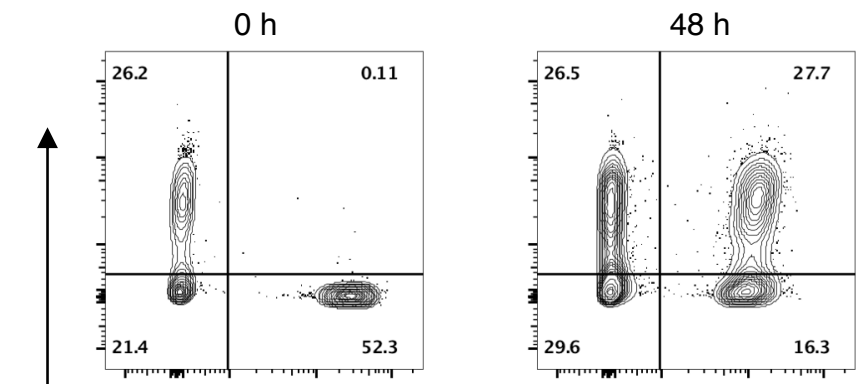

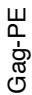

W757A

(Jurkat:Jurkat)
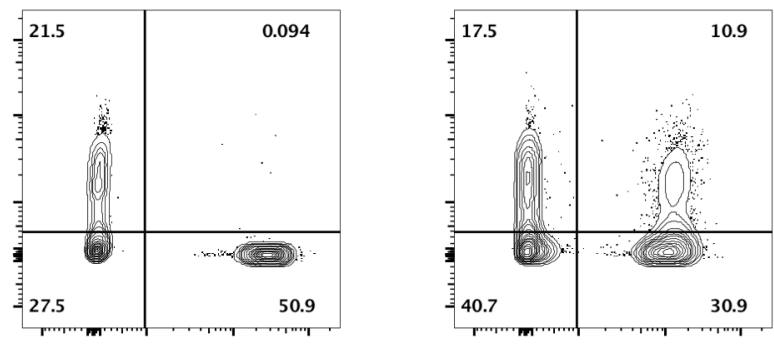

Target Cells (CTFR)

WT

B

(Primary CD4+:Primary CD4+)
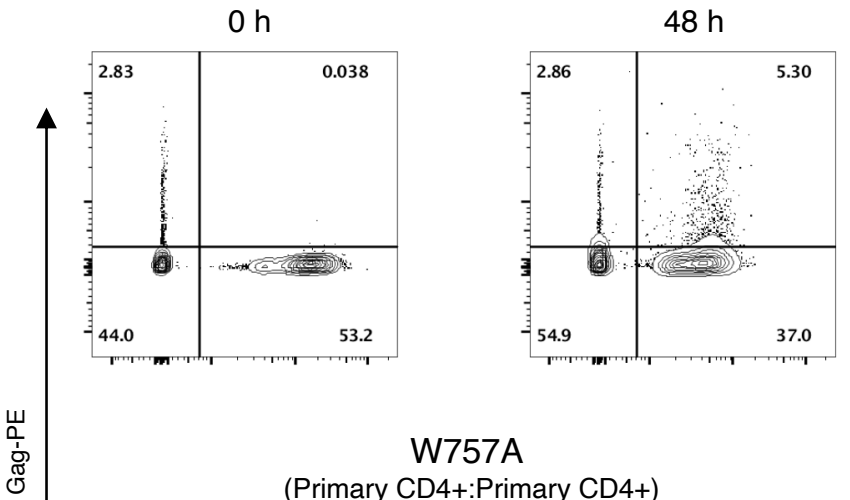

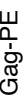

W757A

(Primary CD4+:Primary CD4+)
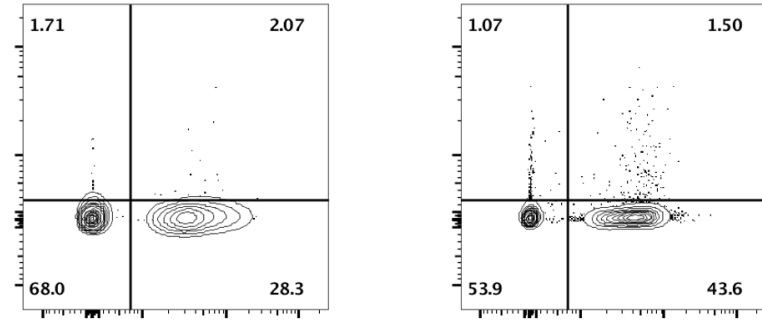

Target Cells (CTFR)

Supplementary Figure 1. EnvCT W757A is defective in T cell - T cell spread. Representative flow cytometry data of cell-cell spread between Jurkat $T$ cells $(\mathbf{A})$ and primary $C D 4^{+} T$ cells $(\mathbf{B})$. The percentage of cells in each quadrant is indicted. 
A

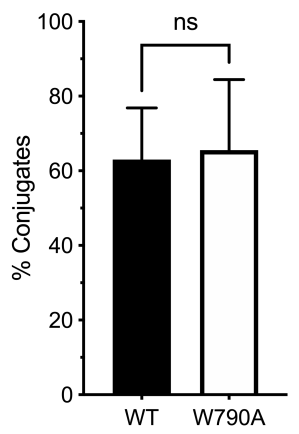

D

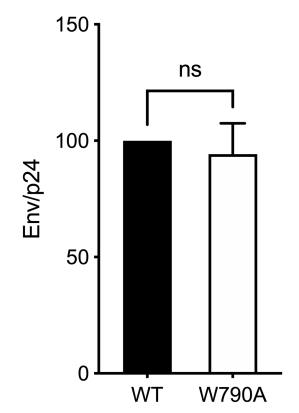

F

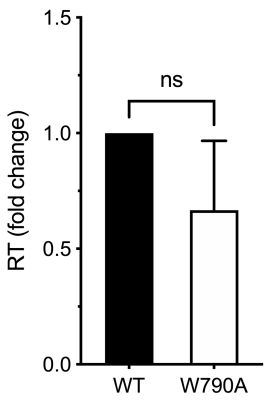

B

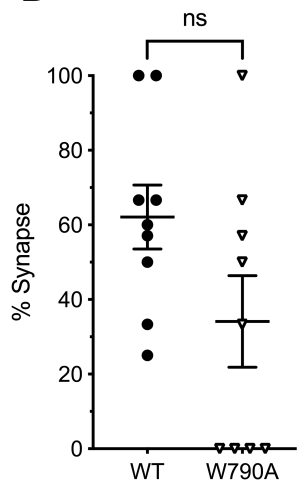

E

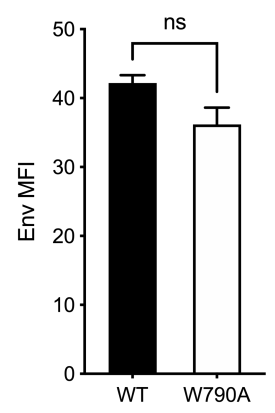

G

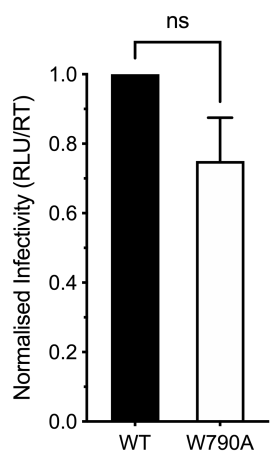

C Intracellular W790A

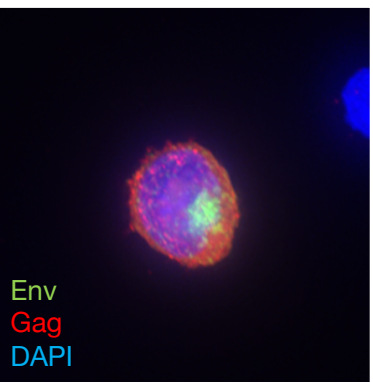

Surface W790A

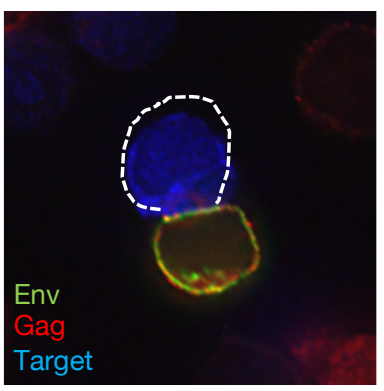

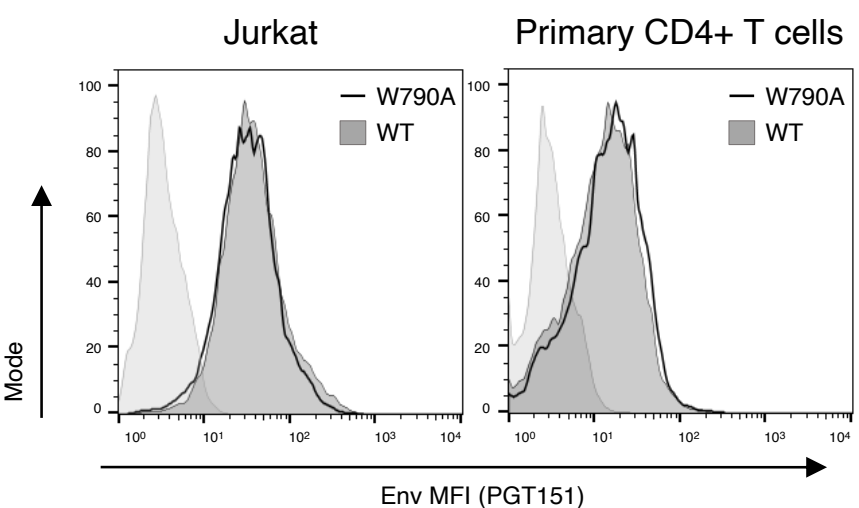

$\mathrm{H}$

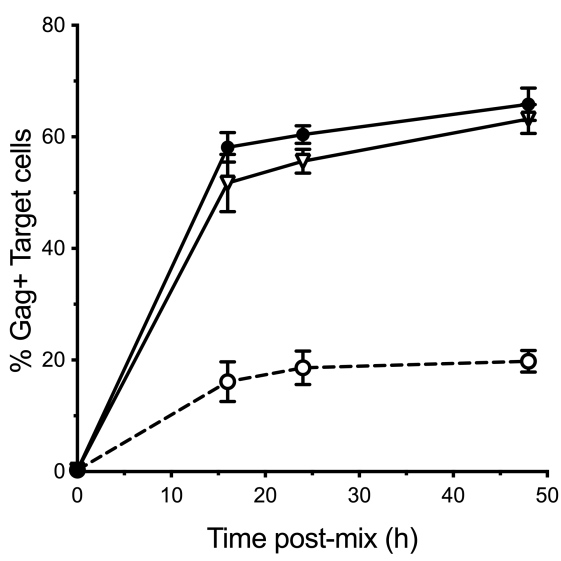

$\rightarrow$ WT

$\rightarrow$ W790A

- W. W757A

Supplementary Figure 2. EnvCT W790A virus does not perturb HIV-1 Env function and viral cell-cell spread. (A) Alanine substitution of the tryptophan at HIV-1 envelope position 790 does not affect the ability of infected Jurkat cells to form conjugates with uninfected target cells. (B-C) W790A infected cells also form synapses to the same extent at WT HIV-1 and result in no defect in both intracellular and surface localization of Env. (D) Quantification of Env (gp120) incorporation into virions produced in Jurkat cells, normalised to Gag (p24) density. (E) Cell surface W790A Envelope (grey) measured by flow cytometry is equal to WT (black) levels. (F-G) Budding of virons from infected cells, and the relative infectivity of virions is equivalent to WT. (H) Viral transfer via cell-cell spread is the same with WT Env and W790A Env, but is significantly defective in W757A Env infection. 
A

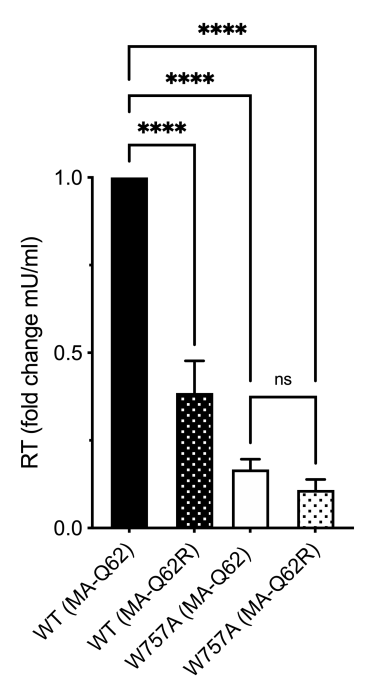

B

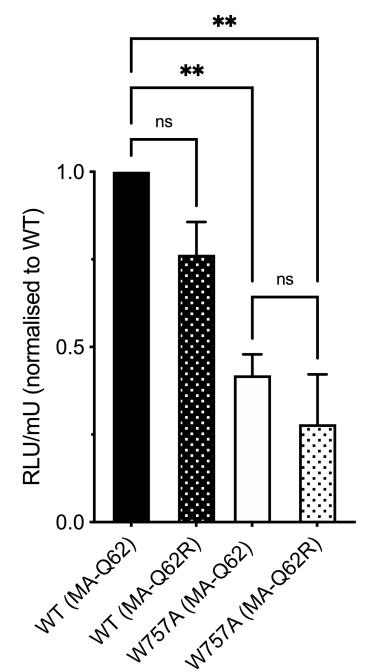

Supplementary Figure 3. Compensatory Matrix mutant (Q62R) is unable to rescue W757A. MA mutation Q62R does not rescue the defect in W757A budding (A) or infectivity (B). Data are the mean and SEM compared using a one way ANOVA (ns, not significant; ${ }^{* *} p<0.01 ;{ }^{* * * *} p<0.0001$ ). 


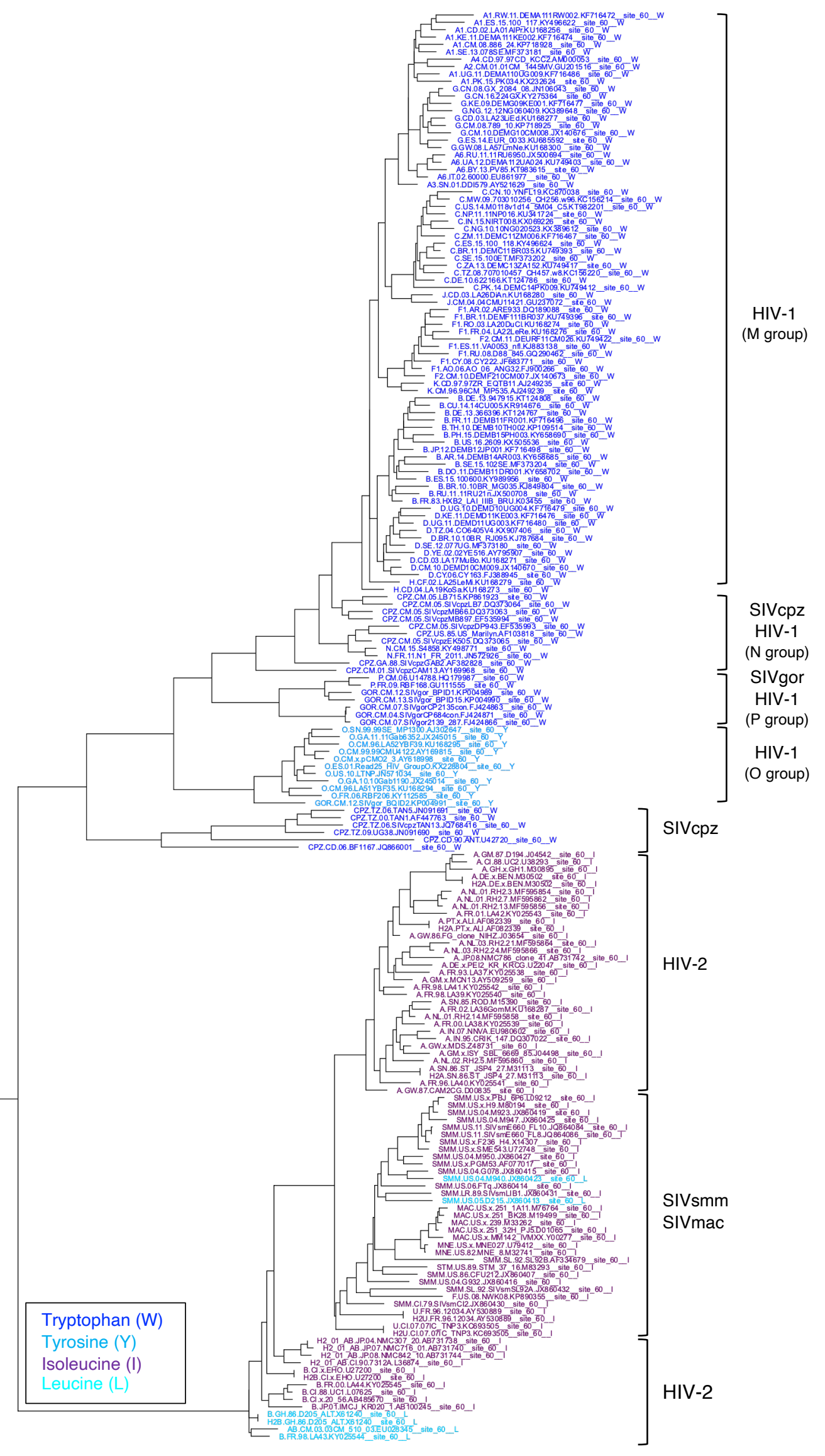

Supplementary Figure 4. Conservation of W757 among HIV-1 M group, SIVcpz, and SIVgor envelope glycoproteins. Phylogenetic analysis of the EnvCT showing amino acid residues found at position 757 (based on 194 consensus envelope sequences from http://www.hiv.lanl.gov/ ) performed using Chromaclade. Tryptophan (W) - dark blue. Tyrosine (Y) - light blue. Isoleucine (I) - purple. Leucine (L) - turquoise. 
A
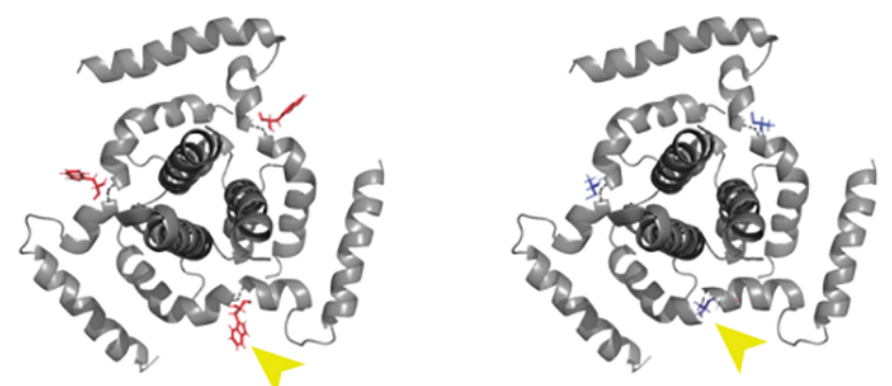

B

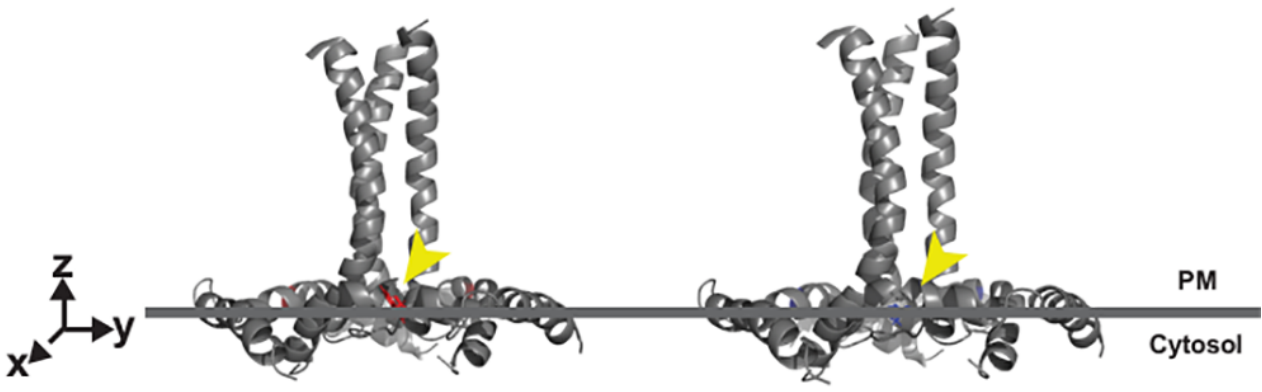

C

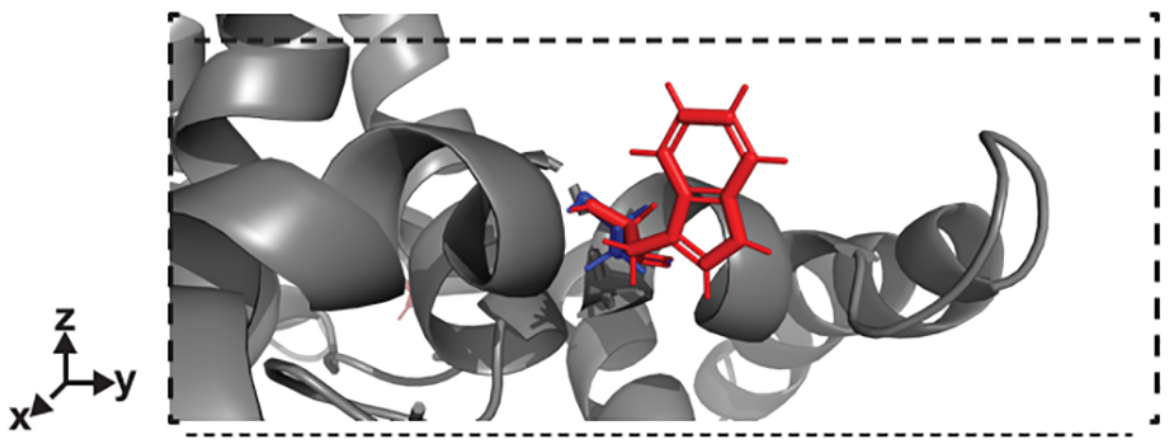

Supplementary Figure 5. W757 within the EnvCT LLP2 helix may stablise the EnvCT baseplate. (A) Top down and (B) side on models of trimeric EnvCT and TMD within the plasma membrane. Trytophan (red) and Alanine (blue) are highlighted in (C). The aromatic side chain of W757 (indicated by the yellow arrow) is predicted to be buried into the membrane, maintaining the EnvCT baseplate conformation, whereas alanine substitution at this position allows for more conformational flexibility in the quaternary structure of the EnvCT baseplate, disrupting the TMD and perhaps MPER/gp120 conformations. 